\title{
35. PALEOMAGNETICS OF TERTIARY SEDIMENTS FROM THE SOUTHWEST ROCKALL PLATEAU, DEEP SEA DRILLING PROJECT LEG 81¹
}

\author{
K. Krumsiek, Geologisches Institut, Bonn \\ and \\ D. G. Roberts, Institute of Oceanographic Sciences, Wormley
}

\begin{abstract}
Paleomagnetic data are tabulated for DSDP Sites 552, 553, 554, and 555, drilled on the southwest margin of Rockall Plateau. No interpretation of these data has been given; because the results provide a particularly valuable record of Paleogene and Neogene magnetostratigraphy they have, however, been provided here for use by the community.
\end{abstract}

\section{INTRODUCTION}

This chapter presents data from the shipboard and post-cruise paleomagnetic studies of sediments and basalts cored on the southwest margin of Rockall Plateau during Leg 81 of the International Phase of Ocean Drilling of the Deep Sea Drilling Project (DSDP). (See Table 1, at end of chapter, and Figs. 1-3.)

The study was intended to extend the results earlier obtained during Leg 48 by Hailwood (1979). It was thought that the paleomagnetic study would provide a useful source of information with which to constrain both the age and eruption history of the lavas thought largely to compose the suite of dipping reflectors. It was also thought that, by providing a more exact chronology, the identification of magnetic polarity reversals might help in understanding the closely spaced events that preceded and heralded the breakup of Greenland and Rockall. Finally, it was hoped that detailed paleomagnetic studies of the late Neogene and Quaternary would contribute to studies of the evolution of North Atlantic paleoceanography and climate (Zimmerman, this volume; Backman, this volume). Unfortunately, time constraints, and changing circumstances in German universities since 1981 which were beyond the control of the senior author (the shipboard paleomagnetist), did not allow preparation of a written report. As the observations are of potentially great value, however, they have been tabulated for future reference, pending a subsequent full report.

\section{PALEOMAGNETIC TECHNIQUES}

Paleomagnetic measurements on Leg 81 were carried out with the equipment installed on Glomar Challenger-a "Digico" computerized spinner magnetometer for measurement of discrete samples having volumes in

\footnotetext{
${ }^{1}$ Roberts, D. G., Schnitker, D., et al., Init. Repts. DSDP, 81: Washington (U.S. Govt. Printing Office).

2 Addresses: (Krumsiek) Geologisches Institut, Friedrick-Wilhelms-Universität, Bonn, Nussallee 8, D5300 Bonn 1, Federal Republic of Germany; (Roberts) Institute of Oceanographic Sciences, Wormley, Godalming, Surrey, United Kingdom (present address: British Petroleum Co. Ltd., Britannic House, Moor Lane, London EC2Y 9BU, United Kingdom).
}

the range of 10 to $20 \mathrm{~cm}^{3}$, and a Schonstedt AF demagnetizer enclosed in a mu-metal shield. A variable speed, reversible, motor-driven system allowed automatic increase of the demagnetizing field to a predetermined peak value, and smooth down to zero. The noise level of the magnetometer was usually in the range of $2 \times 10^{-4}$ $\mathrm{A} / \mathrm{m}$ to $6 \times 10^{-5} \mathrm{~A} / \mathrm{m}$, and satisfactory repeatability of measurements was possible for most samples using an integration time corresponding to $2^{3}$ to $2^{5}$ revolutions.

Additional shipboard measurements were made on HPC cores using the long core spinner. In the case of many samples, further post-cruise studies were carried out in the paleomagnetic laboratories at Bonn and at Zurich to confirm and extend the initial measurements made at sea. At Bonn University the specimens were measured with an UGF 4 spinner magnetometer produced by Geofisika, Brno (CSSR). The sample rotates at 88 revolutions per second; the integration time is 1 or 10 seconds. Demagnetization experiments were carried out with the motor-driven system described above but also incorporating a two-axis tumbling system. During demagnetization, the Earth's magnetic field was compensated down to values $50 \mathrm{nT}$ using Helmholtz coils $1.4 \mathrm{~m}$ in diameter. The magnetic behavior of the RM vector was plotted automatically by means of demagnetization curves (Ir/Io) and Zijderveld diagrams. The paleomagnetic work at Bonn University was limited by the resolution of the spinner magnetometer $\left(2 \times 10^{-5}\right.$ $\mathrm{A} / \mathrm{m}$ ) and by the inconsistency of the specimen.

Post-cruise studies were continued at Zurich University, using a two-axis cryogenic magnetometer for the measurements. The demagnetization experiments were carried out with a Schonstedt AF demagnetizer as has been described. The results were plotted by means of Ir/Io-curves only.

Although the shipboard instruments, the spinner magnetometer, and the AF demagnetizer at Bonn University are still calibrated in the cgs measurement system, the SI metric system is established at Zurich. Since the SI system should be used in all future paleomagnetic work, all data presented here have been converted to this system. 
Table 1. Compilation of paleomagnetic results, Leg 81 .

\begin{tabular}{|c|c|c|c|c|c|c|c|c|}
\hline $\begin{array}{l}\text { Sample } \\
\text { (interval in } \mathrm{cm} \text { ) }\end{array}$ & Decl. & Incl. & $\begin{array}{l}\text { Intensity } \\
(\mathrm{A} / \mathrm{m})\end{array}$ & $\underset{\left(\times 10^{-1} \mathrm{mT}\right)}{\mathrm{AFD}}$ & Decl. & Incl. & $\begin{array}{l}\text { Intensity } \\
(\mathrm{A} / \mathrm{m})\end{array}$ & $(\times 4 \pi$ SI units $)$ \\
\hline \multicolumn{9}{|l|}{ Hole 552} \\
\hline $1-1,73-75$ & 208 & -21 & $3.26 \times 10^{-3}$ & & & & & \\
\hline $1-1,142-144$ & 131 & 6 & $1.82 \times 10^{-3}$ & 480 & 95 & 73 & & \\
\hline $1-2,85-87$ & 280 & 54 & $8.80 \times 10^{-3}$ & 640 & 304 & 50 & $2.75 \times 10^{-3}$ & \\
\hline $1-2,131-133$ & 199 & 58 & $7.72 \times 10^{-3}$ & 320 & 157 & 70 & $2.99 \times 10^{-3}$ & \\
\hline $1-3,11-13$ & 313.3 & 4.1 & $4.76 \times 10^{-4}$ & & & & & \\
\hline $2-1,30-32$ & 34.8 & -41.8 & $1.5 \times 10^{-5}$ & & & & & \\
\hline $2-1,80-82$ & 230 & 67.5 & $3.7 \times 10^{-5}$ & & & & & \\
\hline $2-1,100-102$ & 217 & 38.1 & $9.0 \times 10^{-6}$ & & & & & \\
\hline $3-1,75-77$ & 173.8 & 27.9 & $1.3 \times 10^{-5}$ & & & & & \\
\hline $3-2,75-77$ & 196.2 & 5.0 & $3.0 \times 10^{-6}$ & & & & & \\
\hline $3-3,54-56$ & 15.0 & -54.0 & $8.0 \times 10^{-6}$ & & & & & \\
\hline $3-4,75-77$ & 207 & -21.1 & $3.0 \times 10^{-6}$ & & & & & \\
\hline $3-5,130-132$ & 273.1 & 5.5 & $1.0 \times 10^{-5}$ & & & & & \\
\hline $3-6,75-77$ & 352.3 & -32.2 & $7.0 \times 10^{-6}$ & & & & & \\
\hline $4-1,136-138$ & 90.0 & 33.4 & $2.4 \times 10^{-5}$ & & & & & \\
\hline $4-3,75-77$ & 234.0 & 3.9 & $9.0 \times 10^{-6}$ & & & & & \\
\hline $4-4,68-70$ & 132.0 & 18.0 & $3.6 \times 10^{-5}$ & & & & & \\
\hline $5-1,80-82$ & 261.0 & -8.3 & $3.6 \times 10^{-5}$ & & & & & \\
\hline $5-3,109-111$ & 112.8 & -15.0 & $2.2 \times 10^{-5}$ & & & & & \\
\hline $5-4,20-22$ & 174.3 & 23.0 & $2.7 \times 10^{-5}$ & & & & & \\
\hline $5-5,20-22$ & 204 & 19 & $2.33 \times 10^{-5}$ & & & & & \\
\hline $6-1,19-21$ & 194 & -20 & $1.52 \times 10^{-4}$ & & & & & \\
\hline $6-2,19-21$ & 135 & -22 & $1.05 \times 10^{-3}$ & & & & & \\
\hline $6-3,19-21$ & 141 & -55 & $1.46 \times 10^{-4}$ & & & & & \\
\hline $6-4,19-21$ & 325 & 19 & $1.29 \times 10^{-4}$ & & & & & \\
\hline $7-1,109-111$ & 107 & 14 & $9.12 \times 10^{-5}$ & & & & & \\
\hline $7-2,123-125$ & 188 & 41 & $8.46 \times 10^{-4}$ & 480 & 57 & 1 & $1.89 \times 10^{-5}$ & \\
\hline $7-3,140-142$ & 21 & 21 & $1.07 \times 10^{-3}$ & & & & & \\
\hline $7-4,78-80$ & 33.7 & -46.9 & $1.0 \times 10^{-5}$ & & & & & \\
\hline $8-1,100-102$ & 182.5 & -14.6 & $1.3 \times 10^{-5}$ & & & & & \\
\hline $8-3,100-102$ & 195.0 & 23.0 & $1.3 \times 10^{-5}$ & & & & & \\
\hline $8-4,19-21$ & 207.0 & 37.0 & $1.3 \times 10^{-5}$ & & & & & \\
\hline $8-4,71-73$ & 204.0 & 31.0 & $4.94 \times 10^{-2}$ & 400 & 162 & 51 & $7.46 \times 10^{-5}$ & \\
\hline $9-1,70-72$ & 113.7 & -66.9 & $7.65 \times 10^{-3}$ & 500 & 243 & -70 & $5.5 \times 10^{-4}$ & \\
\hline $9-2,70-72$ & 295.4 & -29.5 & $9.36 \times 10^{-4}$ & & & & & \\
\hline $9-3,70-72$ & 274.1 & -49.6 & $4.49 \times 10^{-3}$ & & & & & \\
\hline $9-4,70-72$ & 1.6 & -64.4 & $2.49 \times 10^{-3}$ & & & & & \\
\hline $9-5,70-72$ & 193.8 & -42.8 & $7.07 \times 10^{-3}$ & & & & & \\
\hline $12-1,60-62$ & 100.1 & -44.7 & $1.06 \times 10^{-3}$ & & & & & \\
\hline $12-1,60-62$ & 104.4 & -63.7 & $1.15 \times 10^{-2}$ & & & & & \\
\hline $12-3,60-62$ & 106.8 & -14.8 & $5.14 \times 10^{-3}$ & & & & & \\
\hline $12-4,60-62$ & 239.4 & -45.0 & $9.09 \times 10^{-3}$ & & & & & \\
\hline $12-5,60-62$ & 340.2 & -61.4 & $9.75 \times 10^{-3}$ & & & & & \\
\hline $12-6,60-62$ & 70.9 & -45.5 & $1.39 \times 10^{-3}$ & & & & & \\
\hline $12-7,48-50$ & 285.5 & 36.0 & $1.07 \times 10^{-2}$ & & & & & \\
\hline $13-1,119-121$ & 213.9 & 69.4 & $2.91 \times 10^{-3}$ & 320 & 308 & 47 & $2.5 \times 10^{-3}$ & \\
\hline $14-1,94-96$ & 336.3 & 71.0 & $3.50 \times 10^{-3}$ & 720 & 23 & 43 & $2.7 \times 10^{-3}$ & \\
\hline $14-2,27-29$ & 22.5 & 71.6 & $2.18 \times 10^{-2}$ & 500 & 239 & 58 & $1.5 \times 10^{-3}$ & \\
\hline $14-3,140-142$ & 281.5 & 74.3 & $6.61 \times 10^{-3}$ & 400 & 345 & 50 & $2.5 \times 10^{-3}$ & \\
\hline $14-2,15-17$ & 65.0 & 61.4 & $2.91 \times 10^{-3}$ & 640 & 169 & 63 & $2.9 \times 10^{-3}$ & \\
\hline $16-1,74-76$ & 150.7 & -56.9 & $5.02 \times 10^{-3}$ & 700 & 156 & -54 & $1.9 \times 10^{-3}$ & \\
\hline $17-1,29-31$ & 334.3 & 49.6 & $9.09 \times 10^{-4}$ & 500 & 252 & -46 & $1.8 \times 10^{-5}$ & \\
\hline $18-1,58-60$ & 267 & -48 & $7.6 \times 10^{-3}$ & 400 & 189 & -36 & $6.8 \times 10^{-3}$ & \\
\hline $18-2,19-21$ & 176.8 & -44.8 & $1.86 \times 10^{-2}$ & 200 & 177 & -50 & $1.2 \times 10^{-2}$ & \\
\hline $21-1,77-79$ & 219 & -38 & $7.2 \times 10^{-3}$ & 320 & 169 & -19 & $1.5 \times 10^{-3}$ & \\
\hline $21-1,86-88$ & 38.3 & -77.2 & $5.01 \times 10^{-3}$ & 320 & 162 & -53 & $7.0 \times 10^{-4}$ & \\
\hline $21-1,117-119$ & 227.9 & -45.6 & $1.15 \times 10^{-2}$ & 320 & 109 & -37 & $1.2 \times 10^{-3}$ & \\
\hline $21-1,134-136$ & 217.9 & -73.3 & $1.87 \times 10^{-2}$ & 400 & 32 & -60 & $1.3 \times 10^{-3}$ & \\
\hline $21-1,142-144$ & 160.3 & -3.9 & $1.44 \times 10^{-2}$ & 320 & 146 & -3 & $2.4 \times 10^{-3}$ & \\
\hline $21-2,5-7$ & 326.7 & -66.3 & $2.34 \times 10^{-2}$ & 480 & 173 & -65 & $4.2 \times 10^{-3}$ & \\
\hline $21-2,15-17$ & 264.1 & 10.5 & $5.83 \times 10^{-3}$ & & & & & \\
\hline $21-2,36-38$ & 4.6 & 55.1 & $2.45 \times 10^{-3}$ & 50 & 10 & 42 & $1.2 \times 10^{-4}$ & \\
\hline $21-2,65-67$ & 261 & -63.8 & $4.85 \times 10^{-2}$ & 240 & 14 & -61 & $8.7 \times 10^{-3}$ & \\
\hline $21-2,85-87$ & 183.9 & -79.6 & $4.84 \times 10^{-2}$ & 240 & 306 & -66 & $6.7 \times 10^{-3}$ & \\
\hline $21-2,104-106$ & 34.1 & -78.3 & $5.40 \times 10^{-2}$ & 320 & 8 & -43 & $2.8 \times 10^{-3}$ & \\
\hline $21-2,110-112$ & 45.2 & 50.4 & $1.22 \times 10^{-2}$ & 150 & 27 & -30 & $6.7 \times 10^{-4}$ & \\
\hline $21-2,129-131$ & 202.8 & -63.9 & $8.00 \times 10^{-2}$ & 240 & 325 & -55 & $6.0 \times 10^{-3}$ & \\
\hline $21-3,5-7$ & 223.5 & -67.9 & $4.61 \times 10^{-2}$ & 320 & 165 & -63 & $2.6 \times 10^{-3}$ & \\
\hline $21-3,25-27$ & 277 & -61 & $6.6 \times 10^{-2}$ & & & & & \\
\hline $21-3,78-80$ & 316 & -67 & $6.9 \times 10^{-1}$ & 480 & 239 & -66 & $1.6 \times 10^{-2}$ & \\
\hline $22-1,59-61$ & 341.4 & -55.5 & 6.66 & 480 & 181 & -64 & $3.7 \times 10^{-1}$ & \\
\hline $22-2,66-68$ & 82.1 & -68.7 & 10.3 & 400 & 173 & -52 & $3.5 \times 10^{-1}$ & \\
\hline $23-2,6-8$ & 336.7 & -55.9 & 6.79 & 400 & 343 & -50 & $3.3 \times 10^{-1}$ & \\
\hline
\end{tabular}


Table 1. (Continued).

\begin{tabular}{|c|c|c|c|c|c|c|c|c|}
\hline $\begin{array}{c}\text { Sample } \\
\text { (interval in } \mathrm{cm} \text { ) }\end{array}$ & Decl. & Incl. & $\begin{array}{l}\text { Intensity } \\
(\mathrm{A} / \mathrm{m})\end{array}$ & $\underset{\left(\times 10^{-1} \mathrm{mT}\right)}{\mathrm{AFD}}$ & Decl. & Incl. & $\begin{array}{c}\text { Intensity } \\
(\mathrm{A} / \mathrm{m})\end{array}$ & 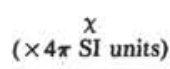 \\
\hline \multicolumn{9}{|l|}{ Hole 552A } \\
\hline $1-1,47-49$ & 291 & 67 & $1.83 \times 10^{-2}$ & & & & & \\
\hline $1-1,80-82$ & 27 & 18 & $1.87 \times 10^{-2}$ & & & & & \\
\hline $1-2,93-95$ & 316 & 58 & $1.77 \times 10^{-2}$ & & & & & \\
\hline $3-1,115-117$ & 55 & 68 & $1.28 \times 10^{-2}$ & & & & & \\
\hline $3-2,115-117$ & 254 & 65 & $4.13 \times 10^{-2}$ & & & & & \\
\hline $3-3,135-137$ & 253 & 57 & $2.50 \times 10^{-2}$ & & & & & \\
\hline $4-1,117-119$ & 342 & 29 & $8.91 \times 10^{-2}$ & 700 & 350 & 20 & $6.62 \times 10^{-2}$ & \\
\hline $4-2,115-117$ & 316 & 21 & $7.32 \times 10^{-3}$ & 700 & 281 & 55 & $2.86 \times 10^{-3}$ & \\
\hline $4-3,113-115$ & 249 & 17 & $9.01 \times 10^{-3}$ & & & & & \\
\hline $5-1,53-55$ & 32 & 61 & $2.45 \times 10^{-2}$ & 500 & 40 & 56 & $8.24 \times 10^{-3}$ & \\
\hline $5-2,53-55$ & 27 & 72 & $4.33 \times 10^{-3}$ & 700 & 246 & -3 & $1.62 \times 10^{-3}$ & \\
\hline $5-3,14-16$ & 288 & 50 & $8.35 \times 10^{-3}$ & 400 & 312 & -26 & $1.48 \times 10^{-3}$ & \\
\hline $5-4,14-16$ & 266 & -6 & $9.53 \times 10^{-4}$ & & & & & \\
\hline $7-1,145-147$ & 20 & 71 & $2.79 \times 10^{-2}$ & & & & & \\
\hline $7-2,105-107$ & 224 & -80 & $1.39 \times 10^{-4}$ & & & & & \\
\hline $7-3,102-104$ & 70 & 73 & $1.44 \times 10^{-2}$ & & & & & \\
\hline $8-1,142-144$ & 213 & -84 & $1.42 \times 10^{-2}$ & & & & & \\
\hline $8-2,141-143$ & 180 & -35 & $5.8 \times 10^{-4}$ & & & & & \\
\hline $8-3,108-110$ & 272 & -38 & $1.17 \times 10^{-2}$ & & & & & \\
\hline $9-1,136-138$ & 237 & -61 & $3.14 \times 10^{-2}$ & & & & & \\
\hline $9-2,106-108$ & 248 & -57 & $4.19 \times 10^{-3}$ & & & & & \\
\hline $9-3,136-138$ & 195 & -10 & $5.39 \times 10^{-4}$ & & & & & \\
\hline $10-1,125-127$ & 268 & -45 & $5.5 \times 10^{-5}$ & & & & & \\
\hline $10-2,24-26$ & 170 & -15 & $4.55 \times 10^{-3}$ & & & & & \\
\hline $10-3,114-116$ & 57 & 24 & $7.2 \times 10^{-4}$ & & & & & \\
\hline $11-2,104-106$ & 165 & 19 & $9.45 \times 10^{-4}$ & & & & & \\
\hline $11-4,6-8$ & 297 & 15 & $1.30 \times 10^{-3}$ & & & & & \\
\hline $12-2,104-106$ & 112 & 58 & $1.48 \times 10^{-3}$ & & & & & \\
\hline $12-3,93-95$ & 186 & -53 & $1.70 \times 10^{-4}$ & & & & & \\
\hline $14-1,113-115$ & 270 & -87 & $5.90 \times 10^{-4}$ & & & & & \\
\hline $14-3,113-115$ & 220 & 14 & $3.00 \times 10^{-5}$ & & & & & \\
\hline $15-1,113-115$ & 137 & -28 & $1.05 \times 10^{-4}$ & & & & & \\
\hline $15-2,113-115$ & 357 & -10 & $1.50 \times 10^{-5}$ & 80 & 254 & 30 & $1.39 \times 10^{-5}$ & \\
\hline $15-3,113-115$ & 317 & 11 & $1.65 \times 10^{-4}$ & & & & & \\
\hline $16-1,117-119$ & 330 & -15 & $3.63 \times 10^{-5}$ & & & & & \\
\hline $16-2,117-119$ & 115 & -18 & $1.40 \times 10^{-4}$ & & & & & \\
\hline $16-3,106-108$ & 297 & -9 & $3.21 \times 10^{-4}$ & & & & & \\
\hline $17-1,106-108$ & 73 & 62 & $1.03 \times 10^{-4}$ & & & & & \\
\hline $18-2,63-65$ & 181 & -41 & $1.60 \times 10^{-4}$ & & & & & \\
\hline $18-3,63-65$ & 237 & 39 & $1.57 \times 10^{-4}$ & & & & & \\
\hline $19-1,107-109$ & 189 & -49 & $8.00 \times 10^{-5}$ & & & & & \\
\hline $19-2,107-109$ & 200 & -61 & $1.00 \times 10^{-4}$ & & & & & \\
\hline $19-3,107-109$ & 16 & -42 & $2.75 \times 10^{-5}$ & & & & & \\
\hline $20-1,63-65$ & 197 & -73 & $2.96 \times 10^{-3}$ & & & & & \\
\hline $20-2,63-65$ & 69 & 42 & $1.63 \times 10^{-4}$ & & & & & \\
\hline $21-1,130-132$ & 54 & -5 & $1.39 \times 10^{-2}$ & & & & & \\
\hline $21-2,130-132$ & 236 & -50 & $1.20 \times 10^{-4}$ & & & & & \\
\hline $21-3,49-51$ & $\begin{array}{l}290 \\
184\end{array}$ & -14 & $2.45 \times 10^{-5}$ & 160 & 211 & 54 & $7.15 \times 10^{-6}$ & \\
\hline $22-1,130-132$ & 117 & -16 & $1.82 \times 10^{-4}$ & & & & & \\
\hline $22-2,130-132$ & 163 & -26 & $5.00 \times 10^{-5}$ & & & & & \\
\hline $22-3,49-51$ & 111 & -39 & $2.17 \times 10^{-5}$ & 240 & 323 & 44 & $8.10 \times 10^{-6}$ & \\
\hline $23-1,89-91$ & 71 & -50 & $8.27 \times 10^{-5}$ & & & & & \\
\hline $23-2,89-91$ & 261 & -2 & $3.42 \times 10^{-5}$ & & & & & \\
\hline $23-3,89-91$ & 297 & -66 & $1.37 \times 10^{-5}$ & 320 & 297 & 60 & $7.64 \times 10^{-6}$ & \\
\hline $24-1,89-91$ & 30 & 18 & $4.13 \times 10^{-5}$ & 120 & 62 & 37 & $8.08 \times 10^{-6}$ & \\
\hline $24-2,89-91$ & 308 & -53 & $1.10 \times 10^{-4}$ & & & & & \\
\hline $24-2,106-108$ & 263 & -53 & $2.20 \times 10^{-5}$ & & & & & \\
\hline $24-3,89-91$ & 90 & -55 & $1.75 \times 10^{-5}$ & & & & & \\
\hline $25-1,88-90$ & 17 & -39 & $4.04 \times 10^{-5}$ & & & & & \\
\hline $25-2,29-31$ & 189 & 14 & $4.25 \times 10^{-5}$ & 320 & 169 & 60 & $1.08 \times 10^{-5}$ & \\
\hline $25-3,110-112$ & 318 & -37 & $5.61 \times 10^{-5}$ & & & & & \\
\hline $26-1,19-21$ & 126 & 10 & $5.91 \times 10^{-3}$ & & & & & \\
\hline $26-1,39-41$ & 161 & -63 & $9.65 \times 10^{-5}$ & 400 & 317 & -59 & $2.7 \times 10^{-5}$ & \\
\hline $26-1,59-61$ & 231 & -50 & $1.62 \times 10^{-4}$ & & & & & \\
\hline $26-1,79-81$ & 338 & -19 & $9.00 \times 10^{-5}$ & & & & & \\
\hline $26-1,99-101$ & 248 & -1 & $9.00 \times 10^{-5}$ & & & & & \\
\hline $26-1,119-121$ & 78 & 14 & $7.7 \times 10^{-4}$ & & & & & \\
\hline $26-1,139-141$ & 80 & -2 & $6.6 \times 10^{-4}$ & & & & & \\
\hline $26-2,19-21$ & 313 & 15 & $1.4 \times 10^{-4}$ & & & & & \\
\hline $26-2,39-41$ & 99 & -25 & $1.60 \times 10^{-4}$ & & & & & \\
\hline $26-2,59-61$ & 13 & 67 & $6.00 \times 10^{-5}$ & & & & & \\
\hline $26-2,79-81$ & 333 & -68 & $3.15 \times 10^{-5}$ & 240 & 319 & -73 & $2.67 \times 10^{-5}$ & \\
\hline $26-2,119-121$ & 76 & 18 & $2.4 \times 10^{-4}$ & $2+0$ & 19 & & & \\
\hline $26-2,139-141$ & 66 & 14 & $1.2 \times 10^{-4}$ & & & & & \\
\hline $26-3,19-21$ & 76 & -1 & $1.1 \times 10^{-4}$ & & & & & \\
\hline
\end{tabular}


Table 1. (Continued).

\begin{tabular}{|c|c|c|c|c|c|c|c|c|}
\hline $\begin{array}{c}\text { Sample } \\
\text { (interval in } \mathrm{cm} \text { ) }\end{array}$ & Decl. & Incl. & $\begin{array}{l}\text { Intensity } \\
(\mathrm{A} / \mathrm{m})\end{array}$ & $\underset{\left(\times 10^{-1} \mathrm{mT}\right)}{\mathrm{AFD}}$ & Decl. & Incl. & $\begin{array}{c}\text { Intensity } \\
(\mathrm{A} / \mathrm{m})\end{array}$ & $(\times 4 \pi \stackrel{\chi}{\text { SI units })}$ \\
\hline \multicolumn{9}{|l|}{ Hole 552A (Cont.) } \\
\hline $26-3,39-41$ & 35 & 31 & $2.0 \times 10^{-4}$ & & & & & \\
\hline $26-3,59-61$ & 149 & $\begin{array}{r}31 \\
-69\end{array}$ & $3.55 \times 10^{-5}$ & & & & & \\
\hline $26-3,79-81$ & 65 & 21 & $8.00 \times 10^{-5}$ & & & & & \\
\hline $26-3,99-101$ & 91 & -11 & $6.00 \times 10^{-5}$ & & & & & \\
\hline $26-3,119-121$ & 46 & -20 & $4.00 \times 10^{-5}$ & & & & & \\
\hline $26-3,140-142$ & 85 & -36 & $1.10 \times 10^{-4}$ & & & & & \\
\hline $27-1,19-21$ & 75 & -72 & $1.00 \times 10^{-4}$ & & & & & \\
\hline $27-1,39-41$ & 25 & -7 & $9.00 \times 10^{-5}$ & & & & & \\
\hline $27-1,58-60$ & 62 & -25 & $1.00 \times 10^{-4}$ & & & & & \\
\hline $27-1,79-81$ & 42 & -18 & $3.00 \times 10^{-5}$ & & & & & \\
\hline $27-1,101-103$ & 24 & 28 & $5.00 \times 10^{-5}$ & & & & & \\
\hline $27-1,139-141$ & 46 & 39 & $4.00 \times 10^{-5}$ & & & & & \\
\hline $27-2,19-21$ & 199 & -71 & $4.9 \times 10^{-4}$ & & & & & \\
\hline $27-2,39-41$ & 92 & 12 & $2.2 \times 10^{-4}$ & & & & & \\
\hline $27-2,59-61$ & 329 & 48 & $5.92 \times 10^{-5}$ & & & & & \\
\hline $27-2,79-81$ & 280 & 60 & $8.00 \times 10^{-5}$ & & & & & \\
\hline $27-2,99-101$ & 75 & 23 & $3.00 \times 10^{-5}$ & & & & & \\
\hline $27-2,119-121$ & 146 & 70 & $2.70 \times 10^{-4}$ & & & & & \\
\hline $27-2,139-141$ & 117 & 71 & $1.00 \times 10^{-4}$ & & & & & \\
\hline $27-3,19-21$ & 7 & 79 & $5.00 \times 10^{-5}$ & & & & & \\
\hline $27-3,40-42$ & 9 & 15 & $3.00 \times 10^{-5}$ & & & & & \\
\hline $27-3,59-61$ & 214 & 46 & $7.57 \times 10^{-5}$ & & & & & \\
\hline $27-3,79-81$ & 324 & $\begin{array}{l}40 \\
59\end{array}$ & $9.00 \times 10^{-5}$ & & & & & \\
\hline $27-3,99-101$ & 11 & 48 & $3.00 \times 10^{-5}$ & & & & & \\
\hline $27-3,119-121$ & 353 & 43 & $5.00 \times 10^{-5}$ & & & & & \\
\hline \multicolumn{9}{|l|}{$27-3,141-143$} \\
\hline $27-4,11-13$ & 34 & 59 & $6.00 \times 10^{-5}$ & & & & & \\
\hline $28-1,19-21$ & 176 & 13 & $1.00 \times 10^{-4}$ & & & & & \\
\hline $28-1,69-71$ & 313 & -68 & $5.84 \times 10^{-3}$ & & & & & \\
\hline $28-1,89-91$ & 67 & 38 & $1.40 \times 10^{-4}$ & & & & & \\
\hline $28-1,112-114$ & 29 & 54 & $1.10 \times 10^{-4}$ & & & & & \\
\hline $28-1,136-138$ & 209 & -40 & $6.50 \times 10^{-4}$ & & & & & \\
\hline $28-2,11-13$ & 307 & 73 & $5.00 \times 10^{-5}$ & 120 & 288 & 73 & $2.15 \times 10^{-5}$ & \\
\hline $28-2,30-32$ & 43 & 73 & $1.00 \times 10^{-4}$ & & & & & \\
\hline $28-2,49-51$ & 171 & 69 & $7.38 \times 10^{-5}$ & & & & & \\
\hline $28-2,69-71$ & 337 & 63 & $7.00 \times 10^{-5}$ & & & & & \\
\hline $28-2,89-91$ & 341 & 60 & $9.00 \times 10^{-5}$ & & & & & \\
\hline $28-2,112-114$ & 8 & 44 & $1.80 \times 10^{-4}$ & & & & & \\
\hline $28-2,135-137$ & 237 & 81 & $2.10 \times 10^{-4}$ & & & & & \\
\hline $28-3,12-14$ & 241 & 68 & $3.47 \times 10^{-5}$ & 320 & 234 & 67 & $1.66 \times 10^{-5}$ & \\
\hline $28-3,30-32$ & 148 & -12 & $1.00 \times 10^{-4}$ & & & & & \\
\hline $28-3,49-51$ & 164 & -51 & $8.00 \times 10^{-5}$ & & & & & \\
\hline $28-3,69-71$ & 65 & -82 & $1.29 \times 10^{-4}$ & & & & & \\
\hline $28-3,112-114$ & 169 & -54 & $1.10 \times 10^{-4}$ & & & & & \\
\hline $28-3,125-127$ & 228 & -58 & $1.40 \times 10^{-4}$ & & & & & \\
\hline $28-3,146-148$ & 186 & -3 & $3.50 \times 10^{-4}$ & & & & & \\
\hline $28-4,11-13$ & 163 & -45 & $7.00 \times 10^{-5}$ & & & & & \\
\hline $28-4,32-34$ & 13 & -79 & $9.50 \times 10^{-5}$ & & & & & \\
\hline $29-1,142-144$ & 205 & 25 & $2.21 \times 10^{-5}$ & 320 & 238 & -50 & $6.15 \times 10^{-6}$ & \\
\hline $29-2,142-144$ & 50 & -15 & $4.30 \times 10^{-4}$ & & & & & \\
\hline $29-3,142-144$ & 90 & 88 & $6.77 \times 10^{-5}$ & 160 & 78 & 79 & $4.28 \times 10^{-5}$ & \\
\hline $30-1,117-119$ & 146 & 17 & $4.00 \times 10^{-5}$ & & & & & \\
\hline $30-2,117-119$ & 150 & -55 & $3.00 \times 10^{-5}$ & & & & & \\
\hline $30-3,117-119$ & 174 & -32 & $9.00 \times 10^{-5}$ & & & & & \\
\hline $31-1,81-83$ & 286 & -58 & $6.00 \times 10^{-5}$ & & & & & \\
\hline $31-2,49-51$ & 211 & -39 & $5.49 \times 10^{-5}$ & & & & & \\
\hline $31-3,48-50$ & 274 & -68 & $5.26 \times 10^{-5}$ & & & & & \\
\hline $32-1,73-75$ & 160 & -76 & $1.74 \times 10^{-3}$ & & & & & \\
\hline $32-2,73-75$ & 159 & -16 & $3.86 \times 10^{-4}$ & & & & & \\
\hline $32-3,73-75$ & 163 & $\begin{array}{r}-10 \\
26\end{array}$ & $3.65 \times 10^{-4}$ & & & & & \\
\hline $33-1,137-139$ & 176 & 71 & $5.69 \times 10^{-4}$ & 160 & 102 & 13 & $2.27 \times 10^{-5}$ & \\
\hline $33-2,137-139$ & 81 & 53 & $1.46 \times 10^{-3}$ & 320 & $\begin{array}{l}102 \\
155\end{array}$ & 81 & $1.73 \times 10^{-4}$ & \\
\hline $33-3,137-139$ & 91 & 49 & $9.58 \times 10^{-5}$ & & & & & \\
\hline $34-1,110-112$ & $\begin{array}{r}91 \\
110\end{array}$ & 12 & $6.61 \times 10^{-5}$ & & & & & \\
\hline $34-2,110-112$ & 132 & -60 & $1.29 \times 10^{-4}$ & & & & & \\
\hline $35-1,130-132$ & 332 & 13 & $2.61 \times 10^{-5}$ & & & & & \\
\hline $35-2,130-132$ & 174 & 28 & $1.26 \times 10^{-3}$ & & & & & \\
\hline $35-3,130-132$ & 143 & 61 & $2.00 \times 10^{-3}$ & & & & & \\
\hline $36-1,35-37$ & 169 & 44 & $6.66 \times 10^{-5}$ & 320 & 186 & 46 & $2.11 \times 10^{-5}$ & \\
\hline $36-2,35-37$ & 32 & -57 & $1.83 \times 10^{-2}$ & 640 & 358 & -43 & $1.47 \times 10^{-3}$ & \\
\hline $36-3,35-37$ & 131 & -57 & $1.26 \times 10^{-2}$ & & & & & \\
\hline $36-3,135-137$ & 251 & -16 & $1.90 \times 10^{-4}$ & & & & & \\
\hline $37-2,56-57$ & 214 & 73 & $1.31 \times 10^{-2}$ & & & & & \\
\hline $37-2,73-74$ & 170 & 67 & $2.53 \times 10^{-2}$ & & & & & \\
\hline $37-2,118-119$ & 92 & $\begin{array}{r}01 \\
-8\end{array}$ & $4.77 \times 10^{-3}$ & 320 & 338 & -66 & $1.88 \times 10^{-3}$ & \\
\hline
\end{tabular}


Table 1. (Continued).

\begin{tabular}{|c|c|c|c|c|c|c|c|c|}
\hline $\begin{array}{c}\text { Sample } \\
\text { (interval in } \mathrm{cm} \text { ) }\end{array}$ & Decl. & Incl. & $\begin{array}{l}\text { Intensity } \\
(\mathrm{A} / \mathrm{m})\end{array}$ & $\underset{\left(\times 10^{-1} \mathrm{mT}\right)}{\mathrm{AFD}}$ & Decl. & Incl. & $\begin{array}{l}\text { Intensity } \\
(\mathrm{A} / \mathrm{m})\end{array}$ & 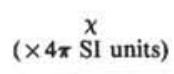 \\
\hline \multicolumn{9}{|l|}{ Hole 552A (Cont.) } \\
\hline $37-3,26-28$ & 351 & -42 & $4.18 \times 10^{-2}$ & & & & & \\
\hline $37-3,56-57$ & 344 & -60 & $2.11 \times 10^{-2}$ & & & & & \\
\hline $37-3,73-75$ & 317 & -73 & $1.91 \times 10^{-2}$ & & & & & \\
\hline $37-3,118-119$ & 328 & -20 & $8.44 \times 10^{-2}$ & & & & & \\
\hline $38-1,135-137$ & 64 & -75 & $2.86 \times 10^{-2}$ & & & & & \\
\hline $38-2,135-137$ & 267 & -62 & $1.88 \times 10^{-2}$ & & & & & \\
\hline $38-3,135-137$ & 169 & -57 & $2.04 \times 10^{-2}$ & & & & & \\
\hline \multicolumn{9}{|l|}{ Hole 553A } \\
\hline $4-2,53-55$ & 119 & -11 & $3.5 \times 10^{-4}$ & & & & & \\
\hline $4-3,85-87$ & 62 & 13 & $2.6 \times 10^{-4}$ & & & & & \\
\hline $4-4,53-55$ & 7 & 13 & $9.1 \times 10^{-4}$ & & & & & \\
\hline $4-5,53-55$ & 112 & 69 & $1.9 \times 10^{-4}$ & & & & & \\
\hline $4-6,53-55$ & 8 & 75 & $1.9 \times 10^{-4}$ & 480 & 29 & 75 & $4.25 \times 10^{-4}$ & \\
\hline $4-7,30-32$ & 219 & -40 & $3.2 \times 10^{-4}$ & & & & & \\
\hline $5-1,91-93$ & 243 & -9 & $2.6 \times 10^{-4}$ & & & & & \\
\hline $5-2,91-93$ & 87 & -1 & $7.0 \times 10^{-4}$ & & & & & \\
\hline $5-3,91-93$ & 347 & -64 & $5.6 \times 10^{-4}$ & & & & & \\
\hline $5-4,91-93$ & 152 & 18 & $4.8 \times 10^{-4}$ & & & & & \\
\hline $5-5,91-93$ & 161 & 5 & $3.3 \times 10^{-4}$ & 240 & 205 & 32 & $7.1 \times 10^{-4}$ & \\
\hline $5-6,91-93$ & 176 & -68 & $5.8 \times 10^{-4}$ & 320 & 147 & 55 & $1.2 \times 10^{-4}$ & \\
\hline $6-1,20-22$ & 204 & 58 & 1.1 & 420 & 256 & 64 & $2.5 \times 10^{-4}$ & \\
\hline $6-3,20-22$ & 143 & 75 & $1.1-4$ & & & & & \\
\hline $6-4,20-22$ & 168 & 62 & $8.7 \times 10^{-4}$ & 640 & 54 & 69 & $2.36 \times 10^{-4}$ & \\
\hline $6-5,15-17$ & 229 & 37 & $6.0 \times 10^{-4}$ & & & & & \\
\hline $6-6,15-17$ & 261 & 18 & $9.6 \times 10^{-3}$ & & & & & \\
\hline $7-1,120-122$ & 119 & -47 & $1.3 \times 10^{-3}$ & & & & & \\
\hline $7-5,37-39$ & 151 & -6 & $3.5 \times 10^{-4}$ & 320 & 193 & -21 & $7.7 \times 10^{-5}$ & \\
\hline $7-5,119-121$ & 132 & -47 & $8.5 \times 10^{-4}$ & & & & $26 \times 10^{-4}$ & \\
\hline $8-1,113-115$ & 225 & 4 & $3.25 \times 10^{-4}$ & 200 & 219 & -42 & $2.6 \times 10^{-4}$ & \\
\hline $8-2,19-21$ & 252 & 13 & $1.2 \times 10^{-3}$ & 600 & 277 & -14 & $7.2 \times 10^{-4}$ & 0.36 \\
\hline $8-3,113-115$ & 163.3 & 77.1 & $4.90 \times 10^{-4}$ & & & & & 2.0 \\
\hline $8-3,136-138$ & 34 & 10 & $9.3 \times 10^{-4}$ & 320 & 77 & -29 & $9.6 \times 10^{-4}$ & \\
\hline $9-1,89-91$ & 245.8 & -41.2 & $4.63 \times 10^{-4}$ & & & & & 0.41 \\
\hline $9-2,88-91$ & 200 & -45 & $1.9 \times 10^{-3}$ & & & & & \\
\hline $9-3,90-92$ & 322 & -73 & $1.1 \times 10^{-3}$ & & & & & \\
\hline $9-4,21-23$ & 101.1 & 52.9 & $4.22 \times 10^{-4}$ & & & & & 0.7 \\
\hline $9-5,55-57$ & 346 & 56 & $5.4 \times 10^{-3}$ & 320 & 311 & 49 & $2.0 \times 10^{-4}$ & 1.95 \\
\hline $9-5,136-138$ & 285.8 & 71.3 & $4.08 \times 10^{-3}$ & & & & & 1.9 \\
\hline $9-6,15-17$ & 138 & -48 & $9.8 \times 10^{-3}$ & 320 & 110 & -56 & $2.7 \times 10^{-3}$ & 2.9 \\
\hline $9-6,57-59$ & 106 & -40 & $5.8 \times 10^{-3}$ & 240 & 81 & -49 & $5.7 \times 10^{-3}$ & 2.2 \\
\hline $10-1,118-119$ & 140.5 & 76.8 & $1.42 \times 10^{-3}$ & & & & & 2.1 \\
\hline $10-2,29-31$ & 28 & 57 & $9.3 \times 10^{-3}$ & 320 & 29 & 70 & $2.3 \times 10^{-3}$ & 2.5 \\
\hline $10-3,88-90$ & 103.8 & 62.2 & $3.01 \times 10^{-2}$ & & & & & 4.4 \\
\hline $10-4,16-18$ & 354 & 69 & $2.0 \times 10^{-2}$ & & & & & 6.5 \\
\hline $10-5,10-12$ & 175 & 68 & $5.1 \times 10^{-3}$ & 480 & 172 & -43 & $1.7 \times 10^{-3}$ & 2.0 \\
\hline $10-6,62-64$ & 166.1 & 76.2 & $1.37 \times 10^{-2}$ & & & & & 3.2 \\
\hline $10-7,42-44$ & 3.8 & -34.4 & $9.04 \times 10^{-3}$ & 320 & 180 & -65 & $1.04 \times 10^{-3}$ & 4.9 \\
\hline $11-1,19-21$ & 229.0 & -13.0 & $1.2 \times 10^{-3}$ & 320 & 353 & -45 & $7.1 \times 10^{-3}$ & 6.2 \\
\hline $11-2,26-28$ & 55.5 & -57.0 & $2.94 \times 10^{-2}$ & & & & & 14.8 \\
\hline $11-3,77-79$ & 253.6 & 57.5 & $1.01 \times 10^{-2}$ & 320 & 80 & 37 & $1.4 \times 10^{-3}$ & 2.6 \\
\hline $11-4,26-28$ & 268.2 & 64.4 & $2.03 \times 10^{-2}$ & & & & & 4.6 \\
\hline $11-5,45-47$ & 310.0 & 67.0 & & & & & & 0.0 \\
\hline $11-6,3-5$ & 217.5 & -26.1 & $3.0 \times 10^{-2}$ & 320 & 65 & -57 & $6.0 \times 10^{-3}$ & 4.1 \\
\hline $11-6,58-60$ & 276.8 & 63.0 & $2.39 \times 10^{-2}$ & 240 & 114 & 33 & & 7.0 \\
\hline $12-1,44-46$ & 239.6 & 62.9 & $2.18 \times 10^{-2}$ & & & & & 5.6 \\
\hline $12-2,25-27$ & 71 & 51 & $1.4 \times 10^{-1}$ & 160 & 97 & 49 & $4.8 \times 10^{-2}$ & \\
\hline $12-3,19-21$ & 36.7 & 71.3 & $4.80 \times 10^{-2}$ & & & & & 12.4 \\
\hline $12-4,19-21$ & 103.5 & 70.9 & $2.70 \times 10^{-2}$ & 160 & 124 & 19 & $1.2 \times 10^{-2}$ & 11.8 \\
\hline $13-1,23-25$ & 26.9 & 69.1 & $6.84 \times 10^{-2}$ & & & & & 10.5 \\
\hline $13-2,99-101$ & 180.1 & 58.8 & $1.51 \times 10^{-2}$ & 160 & 71 & -22 & $5.0 \times 10^{-3}$ & 7.6 \\
\hline $14-1,12-14$ & 224.9 & 62.8 & $3.15 \times 10^{-2}$ & 240 & 147 & -42 & $3.5 \times 10^{-3}$ & 10.5 \\
\hline $14-1,52-54$ & 275 & -45 & $4.0 \times 10^{-2}$ & 480 & 331 & -68 & $1.6 \times 10^{-2}$ & \\
\hline $14-2,107-109$ & 156.7 & 12.4 & $4.30 \times 10^{-3}$ & & & & & 7.9 \\
\hline $14-4,46-48$ & 72.7 & -70.4 & $2.93 \times 10^{-4}$ & & & & & 8.8 \\
\hline $14-5,33-35$ & 201.8 & -25.2 & $2.62 \times 10^{-4}$ & & & & & 7.4 \\
\hline $14-7,30-32$ & 89.3 & -50.8 & $1.25 \times 10^{-2}$ & & & & & 2.0 \\
\hline $15-1,47-49$ & 114.7 & -62.8 & $1.00 \times 10^{-2}$ & & & & & 1.6 \\
\hline $15-2,109-111$ & 286.5 & -36.8 & $1.95 \times 10^{-2}$ & 480 & 83 & 17 & $8.9 \times 10^{-3}$ & 7.3 \\
\hline $15-3,142-144$ & 67.9 & 67.8 & $1.84 \times 10^{-2}$ & 160 & 146 & 30 & $3.5 \times 10^{-2}$ & 16.6 \\
\hline $15-4,63-65$ & 186.9 & 70.9 & $8.17 \times 10^{-2}$ & & & & & 13.0 \\
\hline $18-1,104-106$ & 105.5 & -6.0 & $5.75 \times 10^{-2}$ & 240 & 199 & -12 & $1.9 \times 10^{-2}$ & 9.9 \\
\hline $18-2,37-40$ & 343.3 & 19.0 & $1.45 \times 10^{-2}$ & & & & & 12.7 \\
\hline $19-1,54-56$ & 33.0 & -55 & $1.24 \times 10^{-2}$ & 160 & 309 & -65 & $8.4 \times 10^{-3}$ & 4.8 \\
\hline
\end{tabular}


Table 1. (Continued).

\begin{tabular}{|c|c|c|c|c|c|c|c|c|}
\hline $\begin{array}{c}\text { Sample } \\
\text { (interval in } \mathrm{cm} \text { ) }\end{array}$ & Decl. & Incl. & $\begin{array}{l}\text { Intensity } \\
(\mathrm{A} / \mathrm{m})\end{array}$ & $\underset{\left(\times 10^{-1} \mathrm{mT}\right)}{\mathrm{AFD}}$ & Decl. & Incl. & $\begin{array}{l}\text { Intensity } \\
(\mathrm{A} / \mathrm{m})\end{array}$ & $(\times 4 \pi \stackrel{\chi}{\text { SI units })}$ \\
\hline \multicolumn{9}{|l|}{ Hole 553A (Cont.) } \\
\hline $19-2,38-40$ & 76.2 & 78.2 & $1.76 \times 10^{-2}$ & & & & & 5.0 \\
\hline $19-3,101-103$ & 221.8 & 87.7 & $1.67 \times 10^{-2}$ & & & & & 6.9 \\
\hline $19-4,64-66$ & 118.3 & 81.3 & $4.18 \times 10^{-2}$ & 160 & 256 & 63 & $8.9 \times 10^{-3}$ & 8.2 \\
\hline $20-1,39-41$ & 320.4 & -53.4 & $4.03 \times 10^{-3}$ & & & & & 5.7 \\
\hline $20-2,40-42$ & 310.7 & 59.5 & $6.95 \times 10^{-3}$ & & & & & 3.3 \\
\hline $20-3,42-44$ & 193.6 & 76.1 & $1.17 \times 10^{-2}$ & & & & & 2.8 \\
\hline $20-4,43-44$ & 253.3 & 78.5 & $1.15 \times 10^{-2}$ & 100 & 13.0 & -46.3 & $1.41 \times 10^{-3}$ & 4.1 \\
\hline $20-5,45-47$ & 270.4 & 77.4 & $3.99 \times 10^{-3}$ & 300 & 42.5 & -53.7 & $2.02 \times 10^{-4}$ & 3.0 \\
\hline $20-6,22-24$ & 273.8 & 54.5 & $1.25 \times 10^{-2}$ & & & & & 4.7 \\
\hline $21-1,102-104$ & 321.0 & -43.0 & $2.2 \times 10^{-3}$ & & & & & 2.7 \\
\hline $21-2,100-102$ & 63.0 & -50.0 & $1.1 \times 10^{-3}$ & & & & & 3.1 \\
\hline $21-3,54-56$ & 104.4 & 62.8 & $1.36 \times 10^{-4}$ & & & & & 2.5 \\
\hline $21-4,99-101$ & 122.5 & 46.5 & $3.09 \times 10^{-2}$ & & & & & 6.4 \\
\hline $21-5,13-15$ & 51.6 & 54.4 & $3.17 \times 10^{-2}$ & & & & & 6.0 \\
\hline $22-1,108-110$ & 15.1 & 75.0 & $4.05 \times 10^{-3}$ & 100 & 322.8 & -45.5 & $3.80 \times 10^{-3}$ & 5.2 \\
\hline $22-2,95-97$ & 256.9 & -10.9 & $3.6 \times 10^{-5}$ & & & & & 1.4 \\
\hline $22-3,44-46$ & 184.1 & -15.4 & $2.3 \times 10^{-5}$ & & & & & 1.6 \\
\hline $22-4,101-103$ & 10.3 & -39.5 & $1.16 \times 10^{-3}$ & & & & & 3.2 \\
\hline $22-5,57-59$ & 54.9 & 7.3 & $2.61 \times 10^{-4}$ & & & & & 1.6 \\
\hline $23-1,19-21$ & 107.9 & -64.9 & $3.04 \times 10^{-2}$ & & & & & 6.3 \\
\hline $23-2,19-21$ & 237.0 & 1.6 & $1.56 \times 10^{-3}$ & 400 & 187.9 & -52.9 & $1.94 \times 10^{-2}$ & 3.9 \\
\hline $23-3,19-21$ & 63.1 & -58.7 & $2.69 \times 10^{-2}$ & & & & & 6.9 \\
\hline $23-4,12-14$ & 277.4 & -77.1 & $2.00 \times 10^{-2}$ & 300 & 259.8 & -63.0 & $1.97 \times 10^{-2}$ & 7.4 \\
\hline $23-5,13-15$ & 66.0 & -66.0 & $1.86 \times 10^{-2}$ & & & & & $6-0$ \\
\hline $24-1,14-16$ & 170.1 & -43.2 & $1.67 \times 10^{-2}$ & 300 & 172.6 & -54.9 & $7.22 \times 10^{-3}$ & 6.5 \\
\hline $24-2,63-65$ & 43.7 & 27.9 & $1.07 \times 10^{-2}$ & 250 & 29.0 & -3.6 & $9.44 \times 10^{-4}$ & 3.1 \\
\hline $25-1,72-74$ & 232.2 & -25.4 & $2.04 \times 10^{-2}$ & 250 & 275 & -44.4 & $7.1 \times 10^{-3}$ & 8.7 \\
\hline $25-2,78-80$ & 263.4 & 59.1 & $3.69 \times 10^{-2}$ & & & & & 18.6 \\
\hline $26-2,71-73$ & 60.0 & 78.7 & $4.24 \times 10^{-3}$ & & & & & 9.5 \\
\hline $27-1,105-107$ & 147.2 & 54.5 & $1.26 \times 10^{-2}$ & 300 & 243.0 & -66.4 & $1.12 \times 10^{-3}$ & 11.0 \\
\hline $27-2,10-12$ & 16.4 & 30.0 & $1.91 \times 10^{-2}$ & 100 & 35.9 & -56.6 & $1.95 \times 10^{-2}$ & 18.8 \\
\hline $27-3,11-13$ & 37.2 & 39.1 & $7.05 \times 10^{-4}$ & & & & & 13.3 \\
\hline $27-4,13-15$ & 272.6 & 33.6 & $2.78 \times 10^{-3}$ & 450 & 292.6 & -55.0 & $2.30 \times 10^{-3}$ & 13.5 \\
\hline $27-5,31-33$ & 171.1 & 5.0 & $9.77 \times 10^{-3}$ & & & & & 10.6 \\
\hline $27-6,40-42$ & 22.9 & 17.8 & $1.74 \times 10^{-3}$ & 50 & 0.4 & -53.4 & $9.27 \times 10^{-4}$ & 10.6 \\
\hline $34-1,11-13$ & 88.8 & 54.7 & $4.57 \times 10^{-2}$ & 300 & 28.8 & 53.5 & $5.18 \times 10^{-3}$ & 14.4 \\
\hline $35-1,70-72$ & 211.4 & -34.4 & $1.89 \times 10^{-2}$ & 200 & 192.2 & 65.9 & $2.13 \times 10^{-2}$ & 11.6 \\
\hline $35-1,70-81$ & 92.2 & -58.3 & $1.63 \times 10^{-1}$ & 400 & 99.1 & -61.1 & $1.84 \times 10^{-3}$ & 14.0 \\
\hline $36-1,46-48$ & 64.3 & 40.1 & $1.53 \times 10^{-2}$ & 400 & 61.3 & 52.7 & $6.40 \times 10^{-3}$ & 5.5 \\
\hline $36-1,93-95$ & 359.4 & 64.7 & $1.08 \times 10^{-2}$ & 400 & 295.1 & -50.4 & $2.5 \times 10^{-3}$ & 6.7 \\
\hline $36-2,21-23$ & 324.5 & 44.7 & $1.95 \times 10^{-2}$ & 150 & 343.1 & -34.3 & $2.16 \times 10^{-2}$ & 8.7 \\
\hline $36-3,13-15$ & 31.6 & -83.1 & $7.6 \times 10^{-5}$ & & & & & 2.6 \\
\hline $37-1,25-27$ & 296.3 & 51.9 & $2.94 \times 10^{-4}$ & & & & & 2.9 \\
\hline $37-2,89-91$ & 230.6 & 61.0 & $3.56 \times 10^{-3}$ & 200 & 228.4 & 57.8 & $8.36 \times 10^{-4}$ & 2.6 \\
\hline $37-3,31-33$ & 152.0 & 81.5 & $2.37 \times 10^{-3}$ & 200 & 75.7 & 65.1 & $1.95 \times 10^{-4}$ & 2.7 \\
\hline $37-4,138-140$ & 17.5 & 77.0 & $2.70 \times 10^{-2}$ & 750 & 276.1 & -24.9 & $8.89 \times 10^{-3}$ & 4.2 \\
\hline $38-1,24-26$ & 146.2 & -56.2 & 10.37 & & & & & 123.8 \\
\hline $38-2,36-38$ & 247.8 & -65.7 & 4.45 & 480 & 77 & -65 & $5.22 \times 10^{-1}$ & 98.3 \\
\hline $39-1,90-92$ & 119.7 & -56.2 & 1.54 & 450 & 213.2 & -60.3 & $2.31 \times 10^{-1}$ & 108.6 \\
\hline $40-1,95-97$ & 16.5 & -34.0 & $8.74 \times 10^{-1}$ & 450 & 23.2 & -48.3 & $1.66 \times 10^{-1}$ & 154.7 \\
\hline $40-2,19-21$ & 7.0 & -48.9 & $8.05 \times 10^{-1}$ & 120 & 188 & -47 & $7.29 \times 10^{-1}$ & 285.0 \\
\hline $40-3,124-126$ & 38.9 & 5.4 & 1.00 & 100 & 355.5 & -42.2 & $5.12 \times 10^{-1}$ & 98.3 \\
\hline $41-2,30-32$ & 49.1 & -63.0 & 2.17 & & & & & 91.6 \\
\hline $41-2,128-130$ & 261.7 & -66.8 & 2.15 & 240 & 91 & -63 & $6.02 \times 10^{-1}$ & 65.2 \\
\hline $42-1,112-114$ & 119.6 & -55.4 & 1.83 & 200 & 121.2 & -53.5 & $7.84 \times 10^{-1}$ & 183.8 \\
\hline $42-2,37-39$ & 4.3 & -55.6 & 1.83 & & & & & 92.0 \\
\hline $43-1,80-82$ & 122.2 & -37.2 & 5.14 & 240 & 296 & -49 & 2.50 & 190.7 \\
\hline $43-2,52-54$ & 111.1 & 8.4 & $6.59 \times 10^{-1}$ & 200 & 116.5 & -46.7 & $4.10 \times 10^{-1}$ & 260.4 \\
\hline $43-3,35-37$ & 273.6 & -27.9 & 2.18 & 720 & 67 & -53 & $1.13 \times 10^{-1}$ & 110.0 \\
\hline $46-4,29-31$ & 143.7 & -21.2 & 2.42 & & 145.8 & -22.1 & $2.10 \times 10^{-1}$ & 60.4 \\
\hline $46-5,79-81$ & 146.5 & -21.6 & 4.11 & 240 & 326 & -29 & 3.23 & 74.0 \\
\hline $46-6,5-7$ & 299.1 & -27.1 & 1.67 & 320 & 119 & -23 & 1.00 & 69.1 \\
\hline $47-1,58-60$ & 107.6 & 27.7 & 1.54 & 400 & 296 & -33 & $8.29 \times 10^{-1}$ & 65.0 \\
\hline $47-2,132-134$ & 131.4 & -38.3 & 2.60 & 640 & 314 & -40 & $2.57 \times 10^{-1}$ & 76.4 \\
\hline $47-3,80-82$ & 332.2 & -28.5 & 2.86 & 400 & 158 & -28 & 1.25 & 67.6 \\
\hline $47-4,66-68$ & 297.9 & -37.4 & 1.60 & 240 & 126 & -33 & $8.05 \times 10^{-1}$ & 78.8 \\
\hline $48-1,22-24$ & 336.3 & -50.7 & 3.08 & 480 & 166 & -33 & $6.48 \times 10^{-1}$ & 103.4 \\
\hline $48-2,8-10$ & 210.6 & -54.2 & 4.51 & 120 & 28 & -55 & & 113.6 \\
\hline $48-3,119-121$ & 134.5 & -53.8 & 5.42 & 400 & 322 & -54 & $6.54 \times 10^{-1}$ & 82.0 \\
\hline $48-4,29-31$ & 82.6 & -54.2 & 2.50 & 240 & 254 & -56 & $7.24 \times 10^{-1}$ & 87.7 \\
\hline $48-5,79-81$ & 284.2 & -55.4 & 6.14 & 640 & 83 & -59 & $3.20 \times 10^{-1}$ & 79.2 \\
\hline $48-6,110-112$ & 4.4 & -47.0 & 2.93 & 80 & 181 & -55 & 2.50 & 105.0 \\
\hline $49-1,10-12$ & 220.4 & -49.6 & 2.97 & 240 & 44 & -65 & $6.34 \times 10^{-1}$ & 103.5 \\
\hline $49-2,10-12$ & 133.7 & -56.5 & 5.66 & 240 & 307 & -65 & 2.98 & 87.1 \\
\hline $49-3,10-12$ & 71.7 & -62.6 & 5.97 & 480 & 237 & -59 & $7.23 \times 10^{-1}$ & 77.6 \\
\hline
\end{tabular}


Table 1. (Continued).

\begin{tabular}{|c|c|c|c|c|c|c|c|c|}
\hline $\begin{array}{c}\text { Sample } \\
\text { (interval in } \mathrm{cm} \text { ) }\end{array}$ & Decl. & Incl. & $\begin{array}{c}\text { Intensity } \\
(\mathrm{A} / \mathrm{m})\end{array}$ & $\underset{\left(\times 10^{-1} \mathrm{mT}\right)}{\mathrm{AFD}}$ & Decl. & Incl. & $\begin{array}{l}\text { Intensity } \\
(\mathrm{A} / \mathrm{m})\end{array}$ & $(\times 4 \pi$ SI units $)$ \\
\hline \multicolumn{9}{|l|}{ Hole 553A (Cont.) } \\
\hline $49-4,19-21$ & 148.1 & -59.8 & 5.97 & 640 & 327 & -62 & $5.66 \times 10^{-1}$ & 87.3 \\
\hline $49-5,14-16$ & 216.6 & -61.4 & 6.13 & 240 & 36 & -61 & 4.13 & 83.6 \\
\hline $49-6,49-51$ & 341.6 & -62.0 & 5.22 & 240 & 165 & -61 & 3.77 & 93.5 \\
\hline $49-6,97-99$ & 170.0 & -66.4 & 3.40 & 160 & 181 & -65 & 3.32 & 197.2 \\
\hline $50-1,128-130$ & 281.3 & -52.6 & 2.90 & 480 & 117 & -53 & $2.02 \times 10^{-1}$ & 60.0 \\
\hline $50-2,123-125$ & 122.2 & -48.2 & 3.02 & 240 & 299 & -55 & $8.46 \times 10^{-1}$ & 53.9 \\
\hline $50-3,88-90$ & 299.2 & -48.2 & 1.99 & 400 & 124 & -54 & $1.62 \times 10^{-1}$ & 61.6 \\
\hline $51-1,88-90$ & 358.9 & -48.2 & 2.78 & 80 & 178 & -48 & 2.28 & 72.5 \\
\hline $51-2,63-65$ & 1.2 & -48.1 & 3.11 & 160 & 182 & -48 & 2.27 & 48.8 \\
\hline $51-3,30-32$ & 11.4 & -55.4 & 2.97 & 240 & 188 & -57 & 1.19 & 43.7 \\
\hline $52-1,21-23$ & 108 & -35 & 2.46 & 160 & 118 & -55 & $5.61 \times 10^{-1}$ & 54.0 \\
\hline $52-2,59-61$ & 207 & -51 & 4.72 & 640 & 215 & -50 & $5.76 \times 10^{-1}$ & 69.8 \\
\hline $52-3,24-26$ & 131 & -9 & 1.5 & 320 & 137 & -10 & $8.42 \times 10^{-1}$ & \\
\hline $52-4,41-43$ & 290.1 & -48.4 & 2.77 & 240 & 107 & -49 & 1.62 & 162.4 \\
\hline $53-1,87-89$ & 252.2 & -56.0 & 2.61 & 400 & 85 & -55 & $3.46 \times 10^{-1}$ & 76.5 \\
\hline $53-2,83-85$ & 265.9 & -60.8 & 2.32 & 240 & 74 & -59 & $5.93 \times 10^{-1}$ & 93.6 \\
\hline $53-3,15-17$ & 151.7 & -59.8 & 2.52 & 880 & 29 & -63 & $6.49 \times 10^{-2}$ & 133.1 \\
\hline $53-3,142-144$ & 92.5 & -65.6 & 5.83 & 240 & 289 & -75 & 1.91 & 84.1 \\
\hline $53-4,24-26$ & 276.3 & -61.8 & 3.30 & 160 & 123 & -63 & 1.90 & 62.4 \\
\hline $53-4,63-65$ & 289.3 & -64.0 & 5.76 & 320 & 131 & -62 & 1.04 & 86.3 \\
\hline $54-1,3-5$ & 99.6 & -66.3 & 4.37 & 480 & 248 & -72 & $6.85 \times 10^{-1}$ & 86.1 \\
\hline $54-2,3-5$ & 271.5 & -64.1 & 3.31 & 640 & 83 & -64 & $2.57 \times 10^{-1}$ & 100.4 \\
\hline $54-2,60-62$ & 261.0 & -74.0 & 3.77 & 400 & 267 & -73 & 1.21 & \\
\hline $54-3,119-121$ & 128.8 & -62.4 & 5.09 & 320 & 335 & -62 & 1.25 & 96.1 \\
\hline $54-4,105-107$ & 125.6 & -59.9 & 5.38 & 120 & 317 & -63 & 4.29 & 89.6 \\
\hline $54-5,75-77$ & 143.3 & -61.4 & 4.36 & 480 & 330 & -63 & $8.58 \times 10^{-1}$ & 119.1 \\
\hline $55-1,100-102$ & 155.8 & -30.7 & 2.28 & 240 & 320 & -35 & $3.59 \times 10^{-1}$ & 138.8 \\
\hline $55-2,75-77$ & 288.8 & -53.3 & 13.20 & 400 & 105 & -56 & 3.23 & 145.9 \\
\hline $55-2,140-142$ & 45.0 & -51.6 & 24.61 & 640 & 223 & -54 & 2.90 & 282.0 \\
\hline $55-3,77-79$ & 295.4 & -59.4 & 4.04 & 240 & 121 & -61 & 2.63 & 68.0 \\
\hline $55-4,75-77$ & 8.9 & -13.9 & $7.04 \times 10^{-1}$ & 320 & 177 & -45 & $4.15 \times 10^{-1}$ & 227.8 \\
\hline $55-5,75-77$ & 202.7 & -53.3 & 4.33 & 320 & 41 & -67 & $6.67 \times 10^{-1}$ & 92.0 \\
\hline $55-6,93-95$ & 339.3 & -58.3 & 3.86 & 640 & 159 & -55 & $3.99 \times 10^{-1}$ & 75.9 \\
\hline $56-1,52-54$ & 147.8 & -55.5 & 1.89 & 880 & 338 & -62 & $1.67 \times 10^{-1}$ & 41.2 \\
\hline $56-2,35-37$ & 41.9 & -64.1 & 1.81 & 480 & 228 & -79 & $1.99 \times 10^{-1}$ & 26.5 \\
\hline $56-3,34-36$ & 3.1 & -68.3 & 1.64 & 720 & 169 & -72 & $1.07 \times 10^{-1}$ & 30.4 \\
\hline $57-1,109-111$ & 191.6 & -52.5 & 17.35 & 640 & 15 & -48 & 1.34 & 141.5 \\
\hline $57-2,30-32$ & 226.6 & -46.4 & 5.97 & 480 & 54 & -49 & 1.39 & 71.9 \\
\hline $58-1,43-45$ & 131.1 & -46.8 & 2.00 & 480 & 308 & -51 & $8.35 \times 10^{-1}$ & 29.1 \\
\hline $58-1,133-135$ & 196.0 & -46.0 & 2.64 & 400 & 201 & -52 & $6.24 \times 10^{-1}$ & \\
\hline $58-2,67-69$ & 261.3 & -44.7 & 1.49 & 480 & 75 & -53 & $2.02 \times 10^{-1}$ & 62.8 \\
\hline $58-2,129-131$ & 38.7 & -57.0 & 1.74 & 320 & 206 & -53 & $3.44 \times 10^{-1}$ & 83.2 \\
\hline $59-1,53-55$ & 202.0 & -46.3 & 5.09 & 640 & 26 & -47 & $4.11 \times 10^{-1}$ & 81.1 \\
\hline $59-2,107-109$ & 257.6 & -48.5 & 2.99 & 320 & 59 & -50 & $4.18 \times 10^{-1}$ & 74.8 \\
\hline $59-3,114-116$ & 318.0 & -52.8 & 4.15 & 640 & 134 & -54 & $1.71 \times 10^{-1}$ & 46.4 \\
\hline $59-4,101-103$ & 114.4 & 52.9 & 3.48 & 400 & 298 & -63 & $2.32 \times 10^{-1}$ & 58.5 \\
\hline \multicolumn{9}{|l|}{ Hole 554} \\
\hline $2-3,101-103$ & 77 & -76 & $8.31 \times 10^{-3}$ & & & & & \\
\hline $2-4,131-133$ & 119 & 43 & $9.29 \times 10^{-3}$ & & & & & \\
\hline $2-5,96-98$ & 275 & 3 & $4.22 \times 10^{-4}$ & & & & & \\
\hline $2-6,38-40$ & 354 & 69 & $1.70 \times 10^{-2}$ & & & & & \\
\hline $3-2,136-138$ & 248 & 1 & $2.05 \times 10^{-3}$ & & & & & \\
\hline $3-4,61-63$ & 241 & -53 & $1.86 \times 10^{-2}$ & & & & & \\
\hline $3-5,35-37$ & 6 & -86 & $1.67 \times 10^{-2}$ & & & & & \\
\hline $3-6,40-42$ & 318 & 47 & $1.06 \times 10^{-2}$ & & & & & \\
\hline $3-6,100-102$ & 317 & 27 & $9.36 \times 10^{-3}$ & & & & & \\
\hline $5-2,76-78$ & 170 & 22 & $9.25 \times 10^{-4}$ & & & & & \\
\hline $5-3,135-137$ & 348 & 7 & $3.22 \times 10^{-4}$ & 400 & 319 & 52 & $9.67 \times 10^{-5}$ & \\
\hline $5-5,78-80$ & 186 & 30 & $3.54 \times 10^{-4}$ & & & & & \\
\hline $5-6,78-80$ & 126 & 54 & $4.92 \times 10^{-4}$ & 40 & 324 & 8 & $7.60 \times 10^{-5}$ & \\
\hline $6-5,144-146$ & 90 & 82 & $1.57 \times 10^{-4}$ & & & & & \\
\hline $6-6,91-93$ & 46 & -31 & $1.03 \times 10^{-5}$ & & & & & \\
\hline $7-3,134-136$ & 116 & 24 & $6.96 \times 10^{-5}$ & & & & & \\
\hline $8-4,45-47$ & 153 & 60 & $1.10 \times 10^{-4}$ & & & & & \\
\hline \multicolumn{9}{|l|}{ Hole 554A } \\
\hline $1-1,91-93$ & 184 & 21 & $5.54 \times 10^{-4}$ & & & & & \\
\hline $2-1,110-112$ & 181 & 31 & $5.83 \times 10^{-4}$ & & & & & \\
\hline $2-2,110-112$ & 106 & 75 & $3.59 \times 10^{-3}$ & & & & & \\
\hline $3-1,20-22$ & 184 & -86 & $1.36 \times 10^{-3}$ & 320 & 108 & 54 & $1.46 \times 10^{-4}$ & \\
\hline $3-2,20-22$ & 216 & 63 & $1.61 \times 10^{-3}$ & 480 & 304 & 70 & $8.69 \times 10^{-4}$ & \\
\hline $3-3,20-22$ & 188 & 77 & $2.42 \times 10^{-4}$ & & & & & \\
\hline
\end{tabular}


Table 1. (Continued).

\begin{tabular}{|c|c|c|c|c|c|c|c|c|}
\hline $\begin{array}{c}\text { Sample } \\
\text { (interval in } \mathrm{cm} \text { ) }\end{array}$ & Decl. & Incl. & $\begin{array}{l}\text { Intensity } \\
(\mathrm{A} / \mathrm{m})\end{array}$ & $\underset{\left(\times 10^{-1} \mathrm{mT}\right)}{\mathrm{AFD}}$ & Decl. & Incl. & $\begin{array}{l}\text { Intensity } \\
(\mathrm{A} / \mathrm{m})\end{array}$ & $\stackrel{\chi}{\stackrel{\chi}{\text { SI units })}}$ \\
\hline Hole 554A (Cont.) & & & & & & & & - \\
\hline $3-4,20-22$ & 317 & 26 & $1.49 \times 10^{-3}$ & & & & & \\
\hline $4-2,10-12$ & 277 & 54 & $6.55 \times 10^{-4}$ & & & & & \\
\hline $4-2,30-32$ & 294 & 83 & $9.20 \times 10^{-5}$ & & & & & \\
\hline $4-2,50-52$ & 61 & 73 & $6.50 \times 10^{-3}$ & & & & & \\
\hline $4-2,70-72$ & 290 & 85 & $2.58 \times 10^{-3}$ & & & & & \\
\hline $4-2,95-97$ & 273 & 39 & $5.20 \times 10^{-5}$ & & & & & \\
\hline $4-2,118-120$ & 113 & 89 & $1.91 \times 10^{-4}$ & & & & & \\
\hline $4-2,138-140$ & 190 & 72 & $2.62 \times 10^{-3}$ & & & & & \\
\hline $4-3,23-25$ & 337 & 66 & $6.46 \times 10^{-4}$ & & & & & \\
\hline $4-3,58-60$ & 308 & 57 & $2.76 \times 10^{-3}$ & & & & & \\
\hline $4-3,80-82$ & 340 & 74 & $4.66 \times 10^{-4}$ & & & & & \\
\hline $4-3,118-120$ & 253 & -20 & $5.87 \times 10^{-4}$ & & & & & \\
\hline $4-4,20-22$ & 345 & 48 & $1.70 \times 10^{-4}$ & & & & & \\
\hline $5-1,29-31$ & 193 & 21 & $1.58 \times 10^{-3}$ & & & & & \\
\hline $5-1,70-72$ & 304 & 55 & $9.50 \times 10^{-5}$ & & & & & \\
\hline $5-1,99-101$ & 318 & 3 & $1.59 \times 10^{-3}$ & 400 & 287 & 44 & $3.02 \times 10^{-4}$ & \\
\hline $5-1,130-132$ & 334 & 61 & $5.16 \times 10^{-4}$ & & & & & \\
\hline $5-2,10-12$ & 205 & 22 & $1.40 \times 10^{-5}$ & & & & & \\
\hline $5-2,41-43$ & 32 & 72 & $7.40 \times 10^{-4}$ & & & & & \\
\hline $5-2,80-82$ & 281 & 71 & $1.66 \times 10^{-3}$ & & & & & \\
\hline $5-2,110-112$ & 60 & 53 & $1.96 \times 10^{-3}$ & & & & & \\
\hline $5-2,146-148$ & & & & & & & & \\
\hline $5-3,22-24$ & 85 & 67 & $5.67 \times 10^{-3}$ & & & & & \\
\hline $5-3,52-54$ & 188 & 13 & $1.88 \times 10^{-3}$ & & & & & \\
\hline $5-3,80-82$ & 355 & 59 & $6.86 \times 10^{-4}$ & & & & & \\
\hline $5-3,118-120$ & 289 & 46 & $6.79 \times 10^{-4}$ & & & & & \\
\hline $5-4,15-17$ & 296 & 46 & $6.00 \times 10^{-5}$ & & & & & \\
\hline $5-4,58-60$ & 27 & 61 & $5.13 \times 10^{-2}$ & & & & & \\
\hline $5-4,137-139$ & 357 & 59 & $6.07 \times 10^{-2}$ & & & & & \\
\hline $6-1,86-88$ & 52 & 56 & $4.48 \times 10^{-2}$ & & & & & \\
\hline $6-2,83-85$ & 355 & 64 & $8.28 \times 10^{-2}$ & & & & & \\
\hline $6-2,115-117$ & 19 & 55 & $4.89 \times 10^{-2}$ & & & & & \\
\hline $6-3,29-31$ & 133 & 72 & $6.67 \times 10^{-1}$ & & & & & \\
\hline $7-1,110-112$ & 59 & 56 & $1.34 \times 10^{-1}$ & & & & & \\
\hline $7-2,29-31$ & 113 & -57 & $2.69 \times 10^{-1}$ & 200 & 107 & -55 & $1.25 \times 10^{-1}$ & \\
\hline $7-3,61-63$ & 200 & 64 & $4.13 \times 10^{-1}$ & & & & & \\
\hline $7-4,34-36$ & 99 & -62 & $4.00 \times 10^{-1}$ & & & & & \\
\hline $8-1,36-38$ & 301 & -19 & 1.77 & & & & & \\
\hline $8-1,55-57$ & 346 & -44 & $8.45 \times 10^{-2}$ & 200 & 338 & 49 & $9.28 \times 10^{-3}$ & \\
\hline $8-1,84-86$ & 252 & 9 & 1.79 & & & & & \\
\hline $9-1,110-112$ & 157 & 54 & 2.42 & 150 & 158 & 45 & $7.5 \times 10^{-1}$ & \\
\hline $10-1,66-68$ & 18 & 51 & 3.14 & & & & & \\
\hline $14-1,70-72$ & 16 & -47 & 3.59 & 150 & 11 & -47 & $8.41 \times 10^{-1}$ & \\
\hline \multicolumn{9}{|l|}{ Hole 555} \\
\hline $1-1,131-133$ & 221.7 & 82.9 & $1.2 \times 10^{-2}$ & & & & & 1.5 \\
\hline $1-2,98-100$ & 48.8 & 24.0 & $4.0 \times 10^{-4}$ & & & & & 2.2 \\
\hline $1-2,112-114$ & 224.1 & 8.3 & $7.7 \times 10^{-2}$ & & & & & 3.3 \\
\hline $1-3,89-91$ & 121.5 & 67.5 & $1.7 \times 10^{-2}$ & & & & & 3.1 \\
\hline $2-2,35-37$ & 61 & 62 & $2.1 \times 10^{-2}$ & & & & & \\
\hline $2-4,12-14$ & 91.8 & 62.7 & $2.4 \times 10^{-2}$ & & & & & 2.0 \\
\hline $3-1,15-17$ & 330.9 & 56.3 & $2.4 \times 10^{-3}$ & & & & & 2.0 \\
\hline $3-1,90-92$ & 179 & -55 & $3.3 \times 10^{-3}$ & & & & & \\
\hline $3-3,76-78$ & 205.2 & 57.8 & $1.0 \times 10^{-3}$ & & & & & 0.9 \\
\hline $3-4,74-76$ & 98.7 & 53.7 & $1.4 \times 10^{-4}$ & & & & & \\
\hline $3-5,75-77$ & 201 & -44 & $1.3 \times 10^{-5}$ & & & & & \\
\hline $3-5,18-20$ & & & & & & & & \\
\hline $6-3,126-128$ & 213 & 7 & $8.2 \times 10^{-5}$ & 120 & 206 & 23 & $2.2 \times 10^{-5}$ & \\
\hline $8-5,23-25$ & 19 & 16 & $3.8 \times 10^{-5}$ & 80 & 49 & 16 & $8.5 \times 10^{-6}$ & \\
\hline $9-2,131-133$ & 59 & -25 & $2.1 \times 10^{-5}$ & & & & & \\
\hline $11-3,41-43$ & 23 & -29 & $2.1 \times 10^{-5}$ & & & & & \\
\hline $12-2,99-101$ & 102 & 36 & $6.4 \times 10^{-5}$ & 240 & 294 & -10 & $5.3 \times 10^{-6}$ & \\
\hline $14-1,35-37$ & 225 & -42 & $3.6 \times 10^{-5}$ & 240 & 7 & -12 & $3.0 \times 10^{-5}$ & \\
\hline $15-1,102-104$ & 232 & -13 & $9.0 \times 10^{-6}$ & & & & & \\
\hline $16-4,11-13$ & 258 & -25 & $2.0 \times 10^{-5}$ & & & & & \\
\hline $17-3,32-34$ & 166 & -47 & $2.4 \times 10^{-5}$ & & & & & \\
\hline $18-2,50-52$ & 126 & 70 & $1.6 \times 10^{-5}$ & & & & & \\
\hline $19-2,75-77$ & 105 & -67 & $6.5 \times 10^{-5}$ & & & & & \\
\hline $22-1,135-137$ & 110 & -40 & $2.7 \times 10^{-5}$ & 160 & 120 & -48 & $1.2 \times 10^{-5}$ & \\
\hline $24-1,67-69$ & 137 & -83 & $3.2 \times 10^{-3}$ & 480 & 336 & -66 & $9.7 \times 10^{-4}$ & \\
\hline $24-4,67-69$ & 193 & -23 & $5.6 \times 10^{-4}$ & 480 & 183 & 23 & $1.8 \times 10^{-4}$ & \\
\hline $24-7,35-37$ & 162 & 4 & $1.3 \times 10^{-3}$ & 320 & 152 & 10 & $4.2 \times 10^{-4}$ & \\
\hline $25-3,23-25$ & 339 & -7 & $1.2 \times 10^{-3}$ & & & & & \\
\hline $25-5,24-26$ & 152 & 24 & $1.6 \times 10^{-3}$ & 480 & 123 & 32 & $3.9 \times 10^{-4}$ & \\
\hline
\end{tabular}


Table 1. (Continued).

\begin{tabular}{|c|c|c|c|c|c|c|c|c|c|c|}
\hline $\begin{array}{c}\text { Sample } \\
\text { (interval in } \mathrm{cm} \text { ) }\end{array}$ & Decl. & Incl. & & $\begin{array}{l}\text { Intensity } \\
(\mathrm{A} / \mathrm{m})\end{array}$ & $\underset{\left(\times 10^{-1} \mathrm{mT}\right)}{\mathrm{AFD}}$ & Decl. & Incl. & & $\begin{array}{l}\text { ntensity } \\
(\mathrm{A} / \mathrm{m})\end{array}$ & $(\times 4 \pi$ SI units $)$ \\
\hline \multicolumn{11}{|l|}{ Hole 555 (Cont.) } \\
\hline $26-5,45-47$ & 196 & 44 & 1.5 & $\times 10^{-5}$ & & & & & & \\
\hline $26-6,35-37$ & 73 & 10 & $\begin{array}{l}1.0 \\
1.1\end{array}$ & $\times 10^{-3}$ & 400 & 88 & -7 & 3.1 & $\times 10^{-4}$ & \\
\hline $27-1,16-18$ & 52 & -72 & 9.3 & $\times 10^{-3}$ & +00 & 00 & & & & 3.4 \\
\hline $27-2,31-33$ & 152 & 31 & 3.0 & $\times 10^{-3}$ & 160 & 353 & 28 & 2.5 & $\times 10^{-3}$ & \\
\hline $27-2,95-97$ & 79.9 & -8.1 & 15.45 & & & & & & & 8.5 \\
\hline $27-2,117-119$ & 149 & -13 & 9.8 & $\times 10^{-3}$ & 320 & 126 & 75 & 7.3 & $\times 10^{-3}$ & 8.3 \\
\hline $27-3,13-15$ & 162 & 11 & 5.0 & $\times 10^{-3}$ & 480 & 93 & -52 & 1.6 & $\times 10^{-3}$ & 9.1 \\
\hline $28-1,5-7$ & 213.5 & 61.7 & 1.8 & $\times 10^{-2}$ & & & & & & 10.4 \\
\hline 30, CC $5-7$ & 133.6 & -33.0 & 1.9 & $\times 10^{-2}$ & & & & & & 13.3 \\
\hline $31-1,111-113$ & 95.0 & 33.7 & 1.0 & $\times 10^{-1}$ & & & & & & 25.7 \\
\hline $32-1,86-88$ & 251.0 & 59.9 & 1.4 & $\times 10^{-1}$ & & & & & & \\
\hline $32-2,119-121$ & 224.8 & 74.9 & 1.2 & $\times 10^{-1}$ & & & & & & 21.9 \\
\hline $32-3,136-138$ & 69.8 & 74.3 & 1.6 & $\times 10^{-1}$ & & & & & & 21.6 \\
\hline $32-4,84-86$ & 66.7 & 73.5 & 9.2 & $\times 10^{-2}$ & & & & & & 31.5 \\
\hline $32-5,70-72$ & 319.0 & 10.5 & 2.2 & $\times 10^{-2}$ & & & & & & 27.0 \\
\hline $32-6,14-16$ & 259.9 & 50.8 & 1.9 & $\times 10^{-2}$ & & & & & & 17.8 \\
\hline $33-1,125-127$ & 309.0 & -14.5 & 6.8 & $\times 10^{-2}$ & & & & & & 22.7 \\
\hline $33-2,69-71$ & 328.3 & 73.0 & 8.5 & $\times 10^{-3}$ & & & & & & 14.4 \\
\hline $33-3,103-105$ & 321.7 & 52.7 & 3.2 & $\times 10^{-2}$ & & & & & & 17.8 \\
\hline $33-4,120-104$ & 292.3 & 50.8 & 4.1 & $\times 10^{-2}$ & & & & & & 18.9 \\
\hline $33-5,129-131$ & 104.5 & 40.7 & 4.3 & $\times 10^{-2}$ & & & & & & 9.9 \\
\hline $33-6,27-31$ & 291.6 & 5.1 & 6.5 & $\times 10^{-2}$ & & & & & & 12.9 \\
\hline $34-1,21-23$ & 255.1 & -1.2 & 1.4 & $\times 10^{-4}$ & & & & & & 4.6 \\
\hline $34-2,97-99$ & 87.3 & 5.3 & 1.2 & $\times 10^{-2}$ & & & & & & 11.4 \\
\hline $34-3,99-101$ & 104 & 0 & 7.1 & $\times 10^{-2}$ & 160 & 75 & -25 & 2.6 & $\times 10^{-2}$ & 22.7 \\
\hline $34-4,30-32$ & 291.6 & -55.6 & 1.5 & $\times 10^{-1}$ & Nov & & & & & 15.6 \\
\hline $34-4,60-62$ & 227.3 & 10.3 & 5.9 & $\times 10^{-4}$ & & & & & & 2.9 \\
\hline $34-5,87-89$ & 223.1 & 0.9 & 4.1 & $\times 10^{-2}$ & & & & & & 41.9 \\
\hline $35-1,6-8$ & 270.2 & -13.4 & 7.1 & $\times 10^{-3}$ & & & & & & 5.5 \\
\hline $36-1,35-37$ & 31.7 & 3.9 & 3.7 & $\times 10^{-3}$ & & & & & & 3.4 \\
\hline $36-1,104-106$ & 308.5 & -33.9 & 6.0 & $\times 10^{-3}$ & & & & & & 4.1 \\
\hline $36-2,12-14$ & 256.1 & 3.1 & 2.4 & $\times 10^{-3}$ & & & & & & 2.9 \\
\hline $37-1,77-79$ & $\begin{array}{l}250.1 \\
104\end{array}$ & 38 & $\begin{array}{l}2.4 \\
8.8\end{array}$ & $\times 10^{-2}$ & 480 & 3 & -28 & 1.6 & $\times 10^{-2}$ & $\begin{array}{l}2.7 \\
6.7\end{array}$ \\
\hline $37-1,77-79$ & 273.2 & 55.7 & 8.2 & $\times 10^{-5}$ & & & & & & \\
\hline $37-2,100-102$ & 64.3 & 58.8 & 1.5 & $\times 10^{-2}$ & & & & & & 2.5 \\
\hline $38-1,118-120$ & 177.4 & 14.5 & 4.1 & $\times 10^{-2}$ & & & & & & 16.6 \\
\hline $38-2,122-124$ & 180.3 & 10.4 & 2.5 & $\times 10^{-2}$ & & & & & & 11.8 \\
\hline $38-3,124-126$ & 183.4 & 76.8 & 5.8 & $\times 10^{-4}$ & & & & & & 2.6 \\
\hline $39-1,97-99$ & 231.5 & 66.6 & 2.3 & $\times 10^{-2}$ & & & & & & 13.1 \\
\hline $39-2,109-111$ & 206.0 & 54.1 & 2.3 & $\times 10^{-2}$ & & & & & & 12.9 \\
\hline $39-3,114-116$ & 56.0 & 13.1 & 3.6 & $\times 10^{-2}$ & & & & & & 11.1 \\
\hline $39-4,93-95$ & 350.7 & -18.9 & 4.6 & $\times 10^{-2}$ & & & & & & 13.7 \\
\hline $39-5,76-78$ & 287.5 & -41.6 & 6.7 & $\times 10^{-2}$ & & & & & & 15.1 \\
\hline $39-6,81-83$ & 344.2 & -12.7 & 4.7 & $\times 10^{-2}$ & & & & & & 14.1 \\
\hline $39-7,22-24$ & 21.5 & 77.1 & 3.0 & $\times 10^{-3}$ & & & & & & 5.1 \\
\hline $40-1,26-28$ & 255.4 & -27.6 & 7.6 & $\times 10^{-2}$ & & & & & & 12.4 \\
\hline $40-3,19-21$ & 194.0 & 15.2 & 1.0 & $\times 10^{-3}$ & & & & & & 2.8 \\
\hline $40-4,54-56$ & 3.9 & -58.0 & 1.3 & $\times 10^{-2}$ & & & & & & 6.9 \\
\hline $40-5,35-37$ & 269.6 & 59.6 & 7.9 & $\times 10^{-4}$ & & & & & & 2.7 \\
\hline $40-6,115-117$ & 171.8 & -18.8 & 1.8 & $\times 10^{-2}$ & & & & & & 9.2 \\
\hline $41-1,108-110$ & 240.7 & 5.5 & 7.8 & $\times 10^{-4}$ & & & & & & 3.1 \\
\hline $41-2,104-106$ & 115.0 & -34.4 & 8.3 & $\times 10^{-4}$ & & & & & & 2.2 \\
\hline $41-3,103-105$ & 335.0 & 24.8 & 5.6 & $\times 10^{-3}$ & & & & & & 3.2 \\
\hline $41-4,100-102$ & 328.2 & -58.2 & 2.3 & $\times 10^{-2}$ & & & & & & 11.1 \\
\hline $41-5,10-12$ & 284.9 & 30.8 & 4.3 & $\times 10^{-2}$ & & & & & & 11.6 \\
\hline $42-1,73-75$ & 125.0 & -51.3 & 2.0 & $\times 10^{-2}$ & & & & & & 5.7 \\
\hline $42-2,126-128$ & 12.5 & 14.3 & 1.7 & $\times 10^{-2}$ & & & & & & 11.6 \\
\hline $42-3,97-99$ & 87.1 & -30.0 & 3.5 & $\times 10^{-2}$ & & & & & & 12.0 \\
\hline $42-4,8-10$ & 74.7 & -27.0 & 1.2 & $\times 10^{-2}$ & & & & & & 10.9 \\
\hline $43-1,135-137$ & 15.4 & 19.4 & 9.5 & $\times 10^{-4}$ & & & & & & 2.1 \\
\hline $43-3,91-93$ & 292.0 & -24.5 & 5.8 & $\times 10^{-3}$ & & & & & & 2.9 \\
\hline $43-4,13-15$ & 283.4 & -27.6 & 7.7 & $\times 10^{-3}$ & & & & & & 2.8 \\
\hline $43-5,70-72$ & 328.1 & -15.1 & 1.4 & $\times 10^{-3}$ & & & & & & 2.9 \\
\hline $44-1,48-50$ & 72.2 & 52.4 & 6.8 & $\times 10^{-4}$ & & & & & & 2.6 \\
\hline $44-2,89-91$ & 254.3 & -15.5 & 7.4 & $\times 10^{-4}$ & & & & & & 2.9 \\
\hline $45-1,66-68$ & 266.1 & 0.4 & 7.9 & $\times 10^{-3}$ & & & & & & 5.0 \\
\hline $45-3,82-84$ & 71.7 & 42.4 & 2.9 & $\times 10^{-4}$ & & & & & & 2.9 \\
\hline $46-1,23-25$ & 178.7 & -29.4 & 3.3 & $\times 10^{-2}$ & & & & & & 7.8 \\
\hline $46-2,63-65$ & 333.8 & -60.6 & 1.1 & $\times 10^{-1}$ & & & & & & 28.3 \\
\hline $46-2,95-97$ & 208.7 & -47.4 & 4.7 & $\hat{\times 10^{-2}}$ & & & & & & 9.5 \\
\hline $46-3,88-90$ & 67.9 & -52.2 & 1.1 & $\times 10^{-1}$ & & & & & & 18.6 \\
\hline $46-4,92-94$ & 273.2 & -42.6 & 5.4 & $\times 10^{-2}$ & & & & & & 13.0 \\
\hline $46-5,76-78$ & 28.4 & -57.1 & 1.6 & $\times 10^{-2}$ & & & & & & 4.7 \\
\hline $46-6,40-42$ & 321.4 & -61.8 & 3.5 & $\times 10^{-3}$ & & & & & & 2.1 \\
\hline $47-1,144-146$ & 207.5 & -57.5 & 6.2 & $\times 10^{-4}$ & & & & & & 2.0 \\
\hline
\end{tabular}


Table 1. (Continued).

\begin{tabular}{|c|c|c|c|c|c|c|c|c|c|}
\hline $\begin{array}{c}\text { Sample } \\
\text { (interval in } \mathrm{cm} \text { ) }\end{array}$ & Decl. & Incl. & & $\begin{array}{l}\text { Intensity } \\
(\mathrm{A} / \mathrm{m})\end{array}$ & $\underset{\left(\times 10^{-1} \mathrm{mT}\right)}{\mathrm{AFD}}$ & Decl. & Incl. & $\begin{array}{l}\text { Intensity } \\
(\mathrm{A} / \mathrm{m})\end{array}$ & $(\times 4 \pi \stackrel{\chi}{\text { SI units })}$ \\
\hline \multicolumn{10}{|l|}{ Hole 555 (Cont.) } \\
\hline $47-2,126-128$ & 67.9 & 71.6 & 1.4 & $\times 10^{-4}$ & & & & & 1.9 \\
\hline $47-3,31-33$ & 316.3 & 74.4 & 3.6 & $\times 10^{-4}$ & & & & & 2.5 \\
\hline $47-4,71-73$ & 358.4 & 77.6 & 2.4 & $\times 10^{-4}$ & & & & & 2.4 \\
\hline $48-1,103-105$ & 112 & 53 & 9.0 & $\times 10^{-4}$ & 240 & 19 & 54 & $1.9 \times 10^{-4}$ & 2.4 \\
\hline $48-2,7-9$ & 341.5 & 43.8 & 1.4 & $\times 10^{-3}$ & & & & & 2.4 \\
\hline $48-3,96-98$ & 110.3 & -41.5 & 2.7 & $\times 10^{-2}$ & & & & & 5.2 \\
\hline $48-4,95-97$ & 94.2 & -60.2 & 1.1 & $\times 10^{-2}$ & & & & & 12.5 \\
\hline $51-1,26-28$ & 47.4 & -16.4 & 4.0 & $\times 10^{-3}$ & & & & & 9.5 \\
\hline $52-1,102-104$ & 350.7 & -34.0 & 3.8 & $\times 10^{-4}$ & & & & & 2.2 \\
\hline $52-3,76-78$ & 151.4 & -37.2 & 2.3 & $\times 10^{-3}$ & & & & & 13.1 \\
\hline $53-1,93-95$ & 138.8 & -45.1 & 6.8 & $\times 10^{-4}$ & & & & & 2.4 \\
\hline $53-2,61-63$ & 65.0 & 2.9 & 9.7 & $\times 10^{-4}$ & & & & & 2.6 \\
\hline $53-3,33-35$ & 267.5 & 37.9 & 2.6 & $\times 10^{-3}$ & & & & & 2.2 \\
\hline $53-4,114-116$ & 225.9 & -3.8 & 3.1 & $\times 10^{-3}$ & & & & & 2.4 \\
\hline $53-6,70-72$ & 247.2 & 42.5 & 1.0 & $\times 10^{-3}$ & & & & & 2.2 \\
\hline $54-4,85-87$ & 269.6 & 20.0 & 1.8 & $\times 10^{-3}$ & & & & & 2.5 \\
\hline $54-2,122-124$ & 289 & -13 & 9.1 & $\times 10^{-4}$ & 320 & 361 & -45 & $2.8 \times 10^{-4}$ & 2.5 \\
\hline $54-3,97-99$ & 343.5 & -9.5 & 1.2 & $\times 10^{-3}$ & & & & & 2.5 \\
\hline $54-4,77-79$ & 173 & -3 & 2.0 & $\times 10^{-3}$ & & & & & 3.5 \\
\hline $54-5,95-97$ & 254.3 & 4.4 & 6.7 & $\times 10^{-3}$ & & & & & 7.2 \\
\hline $54-6,52-54$ & 207.9 & -29.1 & 1.1 & $\times 10^{-3}$ & & & & & 5.4 \\
\hline $55-1,59-61$ & 118 & -12 & 2.7 & $\times 10^{-3}$ & & & & & 5.7 \\
\hline $56-1,121-123$ & 273 & 10 & 6.7 & $\times 10^{-3}$ & 480 & 286 & 0 & $2.2 \times 10^{-3}$ & 3.2 \\
\hline $56-2,47-49$ & 90 & -49 & 1.1 & $\times 10^{-2}$ & & & & & 4.9 \\
\hline $56-3,53-55$ & 328.0 & -43.7 & 2.1 & $\times 10^{-2}$ & & & & & 5.4 \\
\hline $56-4,114-116$ & 180.1 & -5.1 & 1.5 & $\times 10^{-2}$ & & & & & 7.0 \\
\hline $56-5,43-45$ & 176.1 & -3.8 & 1.7 & $\times 10^{-2}$ & & & & & 6.4 \\
\hline $56-6,124-128$ & 61.9 & -20.5 & 1.4 & $\times 10^{-2}$ & & & & & 6.9 \\
\hline $58-1,44-46$ & 183 & 13 & 3.2 & $\times 10^{-4}$ & 320 & 190 & -28 & $5.6 \times 10^{-5}$ & 0.2 \\
\hline $58-2,133-135$ & 110.6 & 61.5 & 5.6 & $\times 10^{-4}$ & & & & & 1.8 \\
\hline $58-3,128-130$ & 286.2 & 34.8 & 7.9 & $\times 10^{-4}$ & & & & & 2.1 \\
\hline $58-4,98-100$ & 281.1 & -2.3 & 7.7 & $\times 10^{-4}$ & & & & & 1.9 \\
\hline $58-5,26-28$ & 306.4 & 27.4 & 4.3 & $\times 10^{-4}$ & & & & & 1.8 \\
\hline $59-2,23-25$ & 322.2 & 36.2 & 9.0 & $\times 10^{-4}$ & & & & & 2.2 \\
\hline $59-3,55-57$ & 108.4 & 79.7 & 8.7 & $\times 10^{-4}$ & & & & 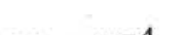 & 2.0 \\
\hline $59-4,89-91$ & 60 & -25 & 1.3 & $\times 10^{-3}$ & 240 & 130 & 25 & $2.2 \times 10^{-4}$ & 1.5 \\
\hline $59-5,129-131$ & 169 & 13 & 3.9 & $\times 10^{-4}$ & 320 & 87 & -27 & $7.9 \times 10^{-5}$ & 1.9 \\
\hline $59-6,89-81$ & 244.9 & 57.6 & 3.0 & $\times 10^{-4}$ & & & & & 1.8 \\
\hline $60-1,80-82$ & 85.3 & 82.3 & 4.3 & $\times 10^{-4}$ & & & & & 2.0 \\
\hline $60-2,10-12$ & 181 & 24 & 3.5 & $\times 10^{-4}$ & 240 & 187 & -8.3 & $4.5 \times 10^{-5}$ & 1.9 \\
\hline $60-3,7-9$ & 308.5 & 68.7 & 2.5 & $\times 10^{-4}$ & & & & & 2.0 \\
\hline $60-4,95-97$ & 263.7 & 57.7 & 3.9 & $\times 10^{-5}$ & & & & & 1.9 \\
\hline $60-5,97-99$ & 208 & 48 & 4.4 & $\times 10^{-4}$ & & & & & 1.9 \\
\hline $60-6,8-10$ & 156 & 35 & 4.5 & $\times 10^{-4}$ & 320 & 166 & 31 & $1.1 \times 10^{-4}$ & 2.1 \\
\hline $60-7,12-14$ & 168 & 1 & 8.5 & $\times 10^{-4}$ & & & & & 1.9 \\
\hline $61-1,32-34$ & 220.6 & 61.4 & 1.9 & $\times 10^{-4}$ & & & & & 2.8 \\
\hline $61-2,9-11$ & 173 & 58 & 1.3 & $\times 10^{-3}$ & 320 & 145 & 25 & $3.2 \times 10^{-4}$ & 2.5 \\
\hline $61-2,36-38$ & 49.8 & 75.5 & 2.5 & $\times 10^{-4}$ & 320 & 140 & 20 & $3.2 \times 10$ & 2.5 \\
\hline $61-3,117-119$ & 144 & 77 & 2.4 & $\times 10^{-4}$ & & & & & 2.4 \\
\hline $61-4,64-66$ & 14.6 & 77.0 & $\begin{array}{l}2.4 \\
1.9\end{array}$ & $\times 10^{-4}$ & & & & & 1.9 \\
\hline $61-5,40-42$ & 127 & -14 & 2.7 & $\times 10^{-4}$ & 400 & 106 & -35 & $4.0 \times 10^{-5}$ & 1.7 \\
\hline $61-6,15-17$ & 259 & -16 & 4.1 & $\times 10^{-4}$ & 400 & 100 & - & $4.0 \wedge 10$ & 2.3 \\
\hline $62-1,89-91$ & 106 & 25 & 2.6 & $\times 10^{-3}$ & 240 & 115 & 61 & $8.4 \times 10^{-4}$ & \\
\hline $62-1,107-109$ & 328.6 & 71.5 & 2.3 & $\times 10^{-3}$ & $2+0$ & Mo & N & & 2.0 \\
\hline $62-2,19-21$ & 40.8 & 70.3 & 7.6 & $\times 10^{-4}$ & & & & & 1.9 \\
\hline $62-3,60-62$ & 11.5 & 81.1 & 4.1 & $\times 10^{-4}$ & & & & & 2.1 \\
\hline $62-4,42-44$ & 314.2 & 67.6 & 2.8 & $\times 10^{-3}$ & & & & & 3.5 \\
\hline $62-5,66-68$ & 352 & -49 & 2.6 & $\times 10^{-3}$ & & & & & 4.5 \\
\hline $63-1,7-9$ & 158 & -42 & 6.2 & $\times 10^{-2}$ & & & & & \\
\hline $63-1,107-119$ & 232.1 & -41.6 & 3.6 & $\times 10^{-2}$ & & & & & 13.8 \\
\hline $64-1,20-22$ & 222 & -43 & 2.0 & $\times 10^{-2}$ & & & & & \\
\hline $64-2,35-37$ & 149.9 & -27.2 & 6.7 & $\times 10^{-2}$ & & & & & 4.2 \\
\hline $64-3,34-36$ & 12.3 & -40.0 & 9.2 & $\times 10^{-3}$ & & & & & 7.5 \\
\hline $64-4,72-74$ & 291.1 & -30.9 & 1.4 & $\times 10^{-2}$ & & & & & 7.5 \\
\hline $64-4,81-83$ & 327.5 & -42.5 & 2.4 & $\times 10^{-2}$ & & & & & 8.3 \\
\hline $64-5,12-14$ & 259.3 & -62.7 & 2.1 & $\times 10^{-2}$ & & & & & 6.5 \\
\hline $65-1,94-96$ & 307.8 & -31.3 & 1.6 & $\times 10^{-2}$ & & & & & 6.1 \\
\hline $65-2,30-32$ & 310.7 & -57.4 & 2.1 & $\times 10^{-2}$ & & & & & 6.2 \\
\hline $66-1,45-47$ & 33.9 & -72.5 & 9.8 & $\times 10^{-3}$ & & & & & 10.1 \\
\hline $67-1,47-49$ & 296.7 & -61.3 & 8.8 & $\times 10^{-3}$ & & & & & 7.8 \\
\hline $67-2,123-125$ & 225.4 & -18.6 & 2.7 & $\times 10^{-2}$ & & & & & 6.9 \\
\hline $67-3,10-12$ & 292.8 & -31.4 & 2.1 & $\times 10^{-2}$ & & & & & 10.4 \\
\hline $67-4,113-115$ & 273.4 & -32.4 & 1.3 & $\times 10^{-1}$ & & & & & 34.0 \\
\hline $68-1,48-50$ & 181.3 & 6.0 & 1.8 & $\times 10^{-3}$ & & & & & 8.1 \\
\hline $68-2,24-26$ & 270.0 & 0.0 & 1.7 & $\times 10^{-2}$ & & & & & 14.2 \\
\hline
\end{tabular}


Table 1. (Continued).

\begin{tabular}{|c|c|c|c|c|c|c|c|c|c|c|}
\hline $\begin{array}{c}\text { Sample } \\
\text { (interval in } \mathrm{cm} \text { ) }\end{array}$ & Decl. & Incl. & & $\begin{array}{l}\text { tensity } \\
(\mathrm{A} / \mathrm{m})\end{array}$ & $\underset{\left(\times 10^{-1} \mathrm{mT}\right)}{\mathrm{AFD}}$ & Decl. & Incl. & & $\begin{array}{l}\text { atensity } \\
(\mathrm{A} / \mathrm{m})\end{array}$ & $\stackrel{\chi}{\text { ( } \times 4 \pi \text { SI units })}$ \\
\hline \multicolumn{11}{|l|}{ Hole 555 (Cont.) } \\
\hline $68-2,56-58$ & 320.5 & -51.5 & 3.0 & & & & & & & 91.2 \\
\hline $68-3,33-35$ & 101.0 & -54.3 & 5.1 & & & & & & & 91.5 \\
\hline $69-1,86-88$ & 105.9 & -59.8 & 3.0 & & & & & & & 89.2 \\
\hline $69-1,85-87$ & 358 & -66 & 3.0 & & 640 & 154 & -63 & 1.1 & $\times 10^{-1}$ & \\
\hline $69-2,61-63$ & 219.5 & -46.3 & 4.9 & & & & & & & 80.4 \\
\hline $69-2,60-62$ & 53 & -44 & 5.3 & & 480 & 128 & -16 & 8.2 & $\times 10^{-2}$ & \\
\hline $69-3,62-64$ & 52.9 & -51.2 & 4.4 & & & & & & & 72.0 \\
\hline $69-3,61-63$ & 205 & -64 & 3.9 & & 320 & 189 & -48 & 9.6 & $\times 10^{-2}$ & \\
\hline $69-4,67$ & 55.5 & -53.1 & 2.8 & & & & & & & 66.2 \\
\hline $69-4,66-68$ & 198 & -76 & 2.5 & & 640 & 199 & -46 & 8.9 & $\times 10^{-2}$ & \\
\hline $69-5,25$ & 260.8 & -8.8 & 1.8 & $\times 10^{-2}$ & & & & & & 12.0 \\
\hline $69-5,27-29$ & 75 & -11 & 3.0 & $\times 10^{-2}$ & 640 & 170 & -44 & 7.5 & $\times 10^{-3}$ & \\
\hline $69-6,33$ & 267.0 & -30.9 & 1.2 & $\times 10^{-2}$ & & & & & & 7.0 \\
\hline $70-1,117$ & 66.8 & -70.4 & 1.4 & & & & & & & 78.5 \\
\hline $70-1,116-118$ & 97 & -42 & 7.6 & $\times 10^{-1}$ & 480 & 169 & -23 & 9.9 & $\times 10^{-3}$ & \\
\hline $70-2,15$ & 35.1 & -59.8 & 1.6 & & & & & & & 85.6 \\
\hline $70-2,14-16$ & 158 & -65 & 1.4 & & 400 & 112 & -39 & 4.4 & $\times 10^{-3}$ & \\
\hline $70-2,40-42$ & 116 & -64 & 2.8 & $\times 10^{-2}$ & 240 & 343 & -56 & 1.0 & $\times 10^{-2}$ & 11.4 \\
\hline $71-1,11-13$ & 89 & -14 & 3.2 & $\times 10^{-2}$ & 880 & 176 & -20 & 1.5 & $\times 10^{-2}$ & \\
\hline $72-1,98-100$ & 323 & 24 & 2.4 & $\times 10^{-2}$ & 480 & 178 & -81 & 9.0 & $\times 10^{-3}$ & 4.9 \\
\hline $72-2,7-9$ & 31 & -53 & 1.2 & & 480 & 357 & -64 & 1.4 & $\times 10^{-2}$ & 72.8 \\
\hline $72-2,31-33$ & 78 & -2 & 2.5 & $\times 10^{-2}$ & 240 & 125 & -37 & 4.7 & $\times 10^{-3}$ & 5.5 \\
\hline $72-3,109-111$ & 113 & -37 & 2.0 & $\times 10^{-2}$ & 240 & 131 & -38 & 3.3 & $\times 10^{-3}$ & 4.4 \\
\hline $73-2,120-122$ & 95 & -19 & 3.9 & $\times 10^{-2}$ & 880 & 178 & -39 & 1.5 & $\times 10^{-2}$ & 7.5 \\
\hline $73-3,68-70$ & 89 & 2 & 2.9 & $\times 10^{-2}$ & 880 & 180 & -28 & 2.3 & $\times 10^{-2}$ & 7.2 \\
\hline $73-4,45-47$ & 106 & -14 & 3.4 & $\times 10^{-2}$ & 160 & 425 & -39 & 1.0 & $\times 10^{-2}$ & 11.4 \\
\hline $73-5,36-38$ & 94 & -35 & 1.7 & $\times 10^{-2}$ & 240 & 184 & -15 & 7.1 & $\times 10^{-3}$ & 7.8 \\
\hline $74-1,22-24$ & 338.6 & -19.4 & 3.3 & $\times 10^{-2}$ & & & & & & 7.9 \\
\hline $74-2,116-118$ & 50 & -36 & 9.3 & $\times 10^{-2}$ & 880 & 1 & -40 & 2.5 & $\times 10^{-3}$ & 6.5 \\
\hline $74-3,47-49$ & 175 & -55 & 1.6 & & 240 & 180 & -58 & 1.2 & $\times 10^{-1}$ & 74.7 \\
\hline $75-1,73-75$ & 182.3 & -52.8 & 2.1 & $\times 10^{-2}$ & & & & & & 3.2 \\
\hline $75-2,36-38$ & 80 & -33 & 8.8 & $\times 10^{-1}$ & 320 & 38 & -40 & 2.6 & $\times 10^{-2}$ & 53.0 \\
\hline $75-2,43-45$ & 52 & -37 & 3.3 & & 480 & 130 & -51 & 1.7 & $\times 10^{-2}$ & 88.8 \\
\hline $76-1,48-50$ & 358 & -50 & 2.0 & & 480 & 2 & -43 & 4.3 & $\times 10^{-2}$ & 63.6 \\
\hline $76-2,23-25$ & 97 & -36 & 3.9 & & 400 & 118 & -31 & 5.2 & $\times 10^{-2}$ & 102.6 \\
\hline $76-2,124-126$ & 23 & -46 & 4.2 & & 880 & 354 & -40 & 1.8 & $\times 10^{-2}$ & 79.8 \\
\hline $76-3,25-27$ & 25 & -42 & 1.7 & & 400 & 36 & -55 & 4.0 & $\times 10^{-2}$ & 65.5 \\
\hline $76-4,78-80$ & 214 & -54 & 3.1 & & 480 & 194 & -40 & 8.0 & $\times 10^{-2}$ & 76.0 \\
\hline $76-5,33-35$ & 88 & -35 & 2.9 & & 480 & 129 & -43 & 2.8 & $\times 10^{-2}$ & 87.6 \\
\hline $77-1,91-93$ & 215 & 29 & 2.2 & $\times 10^{-1}$ & 880 & 178 & -52 & 5.6 & $\times 10^{-2}$ & 8.6 \\
\hline $77-3,67-69$ & 219 & -71 & 1.3 & $\times 10^{-2}$ & 400 & 348 & -49 & 5.2 & $\times 10^{-2}$ & 7.4 \\
\hline $77-5,81-83$ & 240.2 & 41.6 & 2.5 & $\times 10^{-2}$ & & & & & & 4.4 \\
\hline $78-1,67-69$ & 57 & -46 & 5.3 & $\times 10^{-1}$ & 880 & 164 & -57 & 4.5 & $\times 10^{-2}$ & 6.8 \\
\hline $78-3,28-30$ & 52.2 & -63.3 & 1.8 & $\times 10^{-1}$ & & & & & & 6.7 \\
\hline $79-1,34-36$ & 185.4 & -54.5 & 3.7 & $\times 10^{-2}$ & & & & & & 5.5 \\
\hline $79-3,127-129$ & $\begin{array}{l}105.4 \\
69\end{array}$ & -76 & 4.5 & $\times 10^{-3}$ & 880 & 153 & -44 & 9.1 & $\times 10^{-5}$ & 2.2 \\
\hline $80-3,39-41$ & 28 & -72 & 6.5 & $\times 10^{-1}$ & 320 & 259 & -45 & 3.4 & $\times 10^{-2}$ & 96.6 \\
\hline $80-3,125-127$ & 90 & -38 & 4.9 & $\times 10^{-1}$ & 160 & 145 & -64 & 1.2 & $\times 10^{-1}$ & 78.2 \\
\hline $81-1,100-102$ & 82 & -13 & 1.2 & & 320 & 31 & -48 & 1.2 & $\times 10^{-1}$ & 93.4 \\
\hline $81-2,88-90$ & 102 & -21 & 1.4 & & 480 & 148 & -23 & 3.0 & $\times 10^{-2}$ & 63.0 \\
\hline $81-3,86-88$ & 71 & -9 & 1.4 & & 160 & 72 & -33 & 1.9 & $\times 10^{-1}$ & 99.5 \\
\hline $82-1,99-101$ & 146 & -48 & 2.1 & $\times 10^{-2}$ & 140 & 252 & -78 & 1.2 & $\times 10^{-2}$ & 9.4 \\
\hline $82-1,129-131$ & 92 & -36 & 1.4 & & 160 & 130 & -49 & 3.1 & $\times 10^{-1}$ & 93.4 \\
\hline $82-3,102-104$ & 326 & -59 & 4.3 & & 320 & 328 & -53 & 1.9 & $\times 10^{-1}$ & 94 \\
\hline $83-1,30-32$ & 80 & -12 & 7.6 & $\times 10^{-1}$ & 160 & 115 & -43 & 8.1 & $\times 10^{-2}$ & 71.8 \\
\hline $84-1,126-128$ & 67 & -33 & 4.2 & $\times 10^{-3}$ & 720 & 359 & -58 & 9.2 & $\times 10^{-4}$ & 4.4 \\
\hline $84-1,142-144$ & 85 & -14 & 5.0 & $\times 10^{-3}$ & 240 & 107 & -58 & 1.3 & $\times 10^{-3}$ & 6.8 \\
\hline $85-1,47-49$ & 134 & 8 & 1.9 & $\times 10^{-2}$ & 320 & 149 & -13 & 3.8 & $\times 10^{-3}$ & 9.2 \\
\hline $85-2,107-109$ & 220 & -52 & 1.3 & $\times 10^{-1}$ & 480 & 255 & -52 & 1.3 & $\times 10^{-2}$ & 6.0 \\
\hline $86-1,26-28$ & 94 & -49 & 6.2 & $\times 10^{-3}$ & 320 & 220 & -53 & 6.5 & $\times 10^{-4}$ & 7.2 \\
\hline $86-2,44-46$ & 38 & 14 & 5.6 & $\times 10^{-3}$ & 320 & 341 & -39 & 1.7 & $\times 10^{-3}$ & 8.6 \\
\hline $86-3,124-126$ & 147 & -27 & 6.1 & $\times 10^{-3}$ & 480 & 167 & -24 & 1.1 & $\times 10^{-3}$ & 6.5 \\
\hline $87-1,39-41$ & 106 & -45 & 5.1 & $\times 10^{-3}$ & 480 & 178 & -58 & 1.1 & $\times 10^{-3}$ & 7.8 \\
\hline $87-2,10-12$ & 9 & -15 & 7.1 & $\times 10^{-3}$ & 320 & 292 & -56 & 2.7 & $\times 10^{-3}$ & 10.0 \\
\hline $87-3,60-62$ & 108 & -9 & 1.8 & $\times 10^{-2}$ & 320 & 11 & -43 & 3.8 & $\times 10^{-3}$ & 10.0 \\
\hline $87-4,16-18$ & 58 & -28 & 7.2 & $\times 10^{-3}$ & 240 & 28 & -52 & 2.5 & $\times 10^{-3}$ & 6.5 \\
\hline $87-5,31-33$ & 73 & -4 & 1.1 & $\times 10^{-2}$ & 320 & 59 & -39 & 1.8 & $\times 10^{-3}$ & 7.7 \\
\hline $87-5,120-122$ & 41 & -45 & 6.0 & $\times 10^{-3}$ & 240 & 6 & -53 & 3.3 & $\times 10^{-3}$ & 5.0 \\
\hline $88-1,130-132$ & 206 & -79 & 1.8 & $\times 10^{-3}$ & 720 & 229 & -68 & 4.9 & $\times 10^{-4}$ & 3.1 \\
\hline $88-2,30-32$ & 43 & -27 & 7.1 & $\times 10^{-3}$ & 480 & 345 & -49 & 1.3 & $\times 10^{-3}$ & 8.5 \\
\hline $88-3,33-35$ & 44 & 19 & 8.2 & $\times 10^{-3}$ & 640 & 41 & -72 & 1.4 & $\times 10^{-3}$ & 6.2 \\
\hline $88-4,26-28$ & 58 & -17 & 7.8 & $\times 10^{-3}$ & 720 & 72 & -31 & 7.8 & $\times 10^{-4}$ & 6.8 \\
\hline $88-5,4-6$ & 60 & -34 & 2.6 & $\times 10^{-3}$ & 320 & 91 & -66 & 7.8 & $\times 10^{-4}$ & 3.2 \\
\hline $89-1,113-115$ & 81 & -43 & $\begin{array}{l}2.0 \\
1.5\end{array}$ & $\times 10^{-3}$ & 880 & $\begin{array}{r}91 \\
150\end{array}$ & -62 & 2.8 & $\times 10^{-4}$ & 3.0 \\
\hline $90-1,62-64$ & 247 & -72 & 5.7 & & 640 & 14 & -66 & 1.9 & $\times 10^{-4}$ & 146.0 \\
\hline
\end{tabular}


Table 1. (Continued).

\begin{tabular}{|c|c|c|c|c|c|c|c|c|c|}
\hline $\begin{array}{l}\text { Sample } \\
\text { (interval in } \mathrm{cm} \text { ) }\end{array}$ & Decl. & Incl. & & $\begin{array}{l}\text { ntensity } \\
(\mathrm{A} / \mathrm{m})\end{array}$ & $\underset{\left(\times 10^{-1} \mathrm{mT}\right)}{\mathrm{AFD}}$ & Decl. & Incl. & $\begin{array}{l}\text { Intensity } \\
(\mathrm{A} / \mathrm{m})\end{array}$ & $\stackrel{\chi}{\chi}(\times 4 \pi$ SI units) \\
\hline \multicolumn{10}{|l|}{ Hole 555 (Cont.) } \\
\hline $90-1,115-117$ & 107 & -63 & 11.0 & & 480 & 132 & -65 & $1.2 \times 10^{-1}$ & 94.5 \\
\hline $90-2,87-89$ & 64 & -35 & 2.8 & & 120 & 49 & -61 & 1.6 & 111.8 \\
\hline $90-3,86-88$ & 100 & -9 & 2.1 & & 60 & 144 & -22 & $4.1 \times 10^{-1}$ & 125.0 \\
\hline $90-4,69-71$ & 81 & -15 & 1.8 & & 160 & 59 & -59 & $4.2 \times 10^{-1}$ & 100.5 \\
\hline $91-1,43-45$ & 121 & -28 & 1.6 & & 80 & 185 & -62 & $7.0 \times 10^{-1}$ & 129.7 \\
\hline $91-2,97-99$ & 88 & -18 & 3.7 & $\times 10^{-3}$ & 320 & 221 & -49 & $8.3 \times 10^{-4}$ & 7.5 \\
\hline $92-1,94-96$ & 303 & 36 & 3.0 & $\times 10^{-3}$ & 720 & 338 & -38 & $4.0 \times 10^{-4}$ & 5.5 \\
\hline $92-2,86-88$ & 82 & -54 & 1.6 & $\times 10^{-3}$ & 480 & 132 & -67 & $4.3 \times 10^{-4}$ & 5.4 \\
\hline $93-1,80-82$ & 105 & 0. & 1.3 & $\times 10^{-2}$ & 400 & 110 & -37 & $1.9 \times 10^{-3}$ & 8.4 \\
\hline $93-2,53-55$ & 92.2 & 16.1 & 12.7 & & 75 & 134.3 & -88.2 & 1.207 & 7.3 \\
\hline $93-3,80-82$ & 57 & -22 & 1.5 & $\times 10^{-2}$ & 160 & 33 & -43 & $4.7 \times 10^{-3}$ & 7.8 \\
\hline $94-1,88-90$ & 110 & -20 & 6.4 & $\times 10^{-3}$ & 320 & 132 & -33 & $8.2 \times 10^{-4}$ & 4.7 \\
\hline $94-2,24-26$ & 241.1 & -36.1 & 2.4 & $\times 10^{-5}$ & & & & & 2.0 \\
\hline $95-1,30-32$ & 110 & -45 & 4.0 & & 320 & 125 & -45 & & 34.4 \\
\hline $95-1,45-47$ & 96 & -29 & 4.4 & & 240 & 116 & -41 & $6.8 \times 10^{-1}$ & 142.1 \\
\hline $95-1,143-145$ & 145 & -32 & 3.6 & & 880 & 153 & -38 & $3.8 \times 10^{-1}$ & 75.4 \\
\hline $95-2,101-103$ & 33 & -41 & 7.2 & & 720 & 28 & -51 & $7.8 \times 10^{-1}$ & 65.6 \\
\hline $96-1,114-116$ & 69 & -40 & 7.6 & & 640 & 62 & -44 & $2.8 \times 10^{-1}$ & 94.5 \\
\hline $96-2,105-110$ & 194 & -42 & 5.3 & & 480 & 195 & -55 & $1.4 \times 10^{-1}$ & 87.5 \\
\hline $96-3,79-81$ & 155 & -44 & 8.2 & & 640 & 160 & -51 & $1.3 \times 10^{-1}$ & 98.9 \\
\hline $96-4,56-58$ & 118 & -23 & 3.0 & & 480 & 135 & -51 & $1.5 \times 10^{-1}$ & 105.6 \\
\hline $96-5,97-99$ & 355 & -49 & 3.2 & & 720 & 350 & -50 & $3.1 \times 10^{-1}$ & 66.0 \\
\hline $96-6,90-92$ & 342 & -42 & 2.8 & & 480 & 333 & -50 & $1.3 \times 10^{-1}$ & 88.6 \\
\hline $97-1,88-90$ & 295 & -44 & 1.9 & & 880 & 326 & -52 & $3.5 \times 10^{-1}$ & 66.4 \\
\hline $97-3,109-111$ & 98 & -19 & 2.2 & & 720 & 105 & -44 & $5.5 \times 10^{-1}$ & 73.5 \\
\hline $97-5,4-6$ & 64 & -38 & 4.2 & & 480 & 47 & -42 & 2.0 & 63.5 \\
\hline $97-7,9-11$ & 240 & -47 & 2.3 & & 480 & 248 & -50 & 1.3 & 64.3 \\
\hline $98-2,143-145$ & 223 & -40 & 2.4 & & 240 & 224 & -44 & 1.9 & 81.5 \\
\hline $98-4,73-75$ & 223 & -43 & 4.5 & & 720 & 204 & -46 & $4.3 \times 10^{-1}$ & 76.0 \\
\hline $98-5,67-67$ & 49 & -39 & 9.0 & & 320 & 42 & -42 & 1.6 & 97.2 \\
\hline $98-6,18-20$ & 72 & -41 & 7.4 & & 880 & 63 & -42 & $2.3 \times 10^{-1}$ & 64.4 \\
\hline $98-7,32-34$ & 289.4 & 44 & 7.6 & & 500 & 294 & 48 & 1.2 & 68.1 \\
\hline
\end{tabular}

\section{SAMPLING}

In the relatively soft, unconsolidated and semiconsolidated sediments encountered in the upper parts of the holes, paleomagnetic samples were taken by pushing plastic tubes $(2.2 \mathrm{~cm}$ long and $2.5 \mathrm{~cm}$ in diameter) into the split core sections by means of a specially designed plunger supported by an aluminum bridge. The ends of the tubes were sealed with adhesive tape. In the more highly lithified material and basalts encountered at deeper levels, either $2.5 \mathrm{~cm}$-diameter cylindrical samples were drilled from the cores or cube-shaped samples were cut by means of a diamond saw and trimmed to shape using a stainless steel scalpel. In all cases an orientation arrow pointing in the uphole direction was carefully placed on the sample before it was removed from the core. Where inclined bedding was encountered and judged to have resulted either from nonvertical drill penetration or from local movement of portions of the core during drilling, the orientation arrow was aligned perpendicular to the bedding rather than parallel to the core liner.

\section{ASSIGNMENT OF POLARITY}

Unlike conventional piston or gravity cores, DSDP cores are drilled by means of a rotating drill pipe (except in the uppermost, very soft sediments). This process can cause relative rotation (called "disking") between different parts of the core section, with consequent apparent changes in the declination of magnetic remanence. Furthermore, the absolute azimuthal orientation of the cores is not normally measured, and relative azimuthal orientation is not maintained, from one $9.5-\mathrm{m}$ core to the next. For this reason the declination of remanence magnetization is of limited use, except within individual cores in which disking is absent, or in the uppermost few cores at each site, which are commonly drilled without rotation. During Leg 81 a limited number of oriented cores were taken.

Consequently, in this study, magnetic polarity has been assigned on the basis of the inclination of the stable component of remanent magnetization alone. Since all sites are situated at moderate to high latitudes in the Northern Hemisphere, positive magnetic inclinations are taken to signify normal polarity and negative inclinations are taken to indicate reversed polarity. Occasionally, in a series of samples taken from undisturbed portions of the same drill core, polarity reversals detected on the basis of changes in sign of magnetic inclination were corroborated by abrupt changes in declination of approximately $180^{\circ}$.

\section{REFERENCES}

Hailwood, E. A., 1979. Paleomagnetism of late Mesozoic to Holocene sediments from the Bay of Biscay and Rockall Plateau drilled on Leg 48. In Montadert, L., Roberts, D. G., et al., Init. Repts. DSDP, 48:305-369.

Date of Acceptance: June 19, 1984 


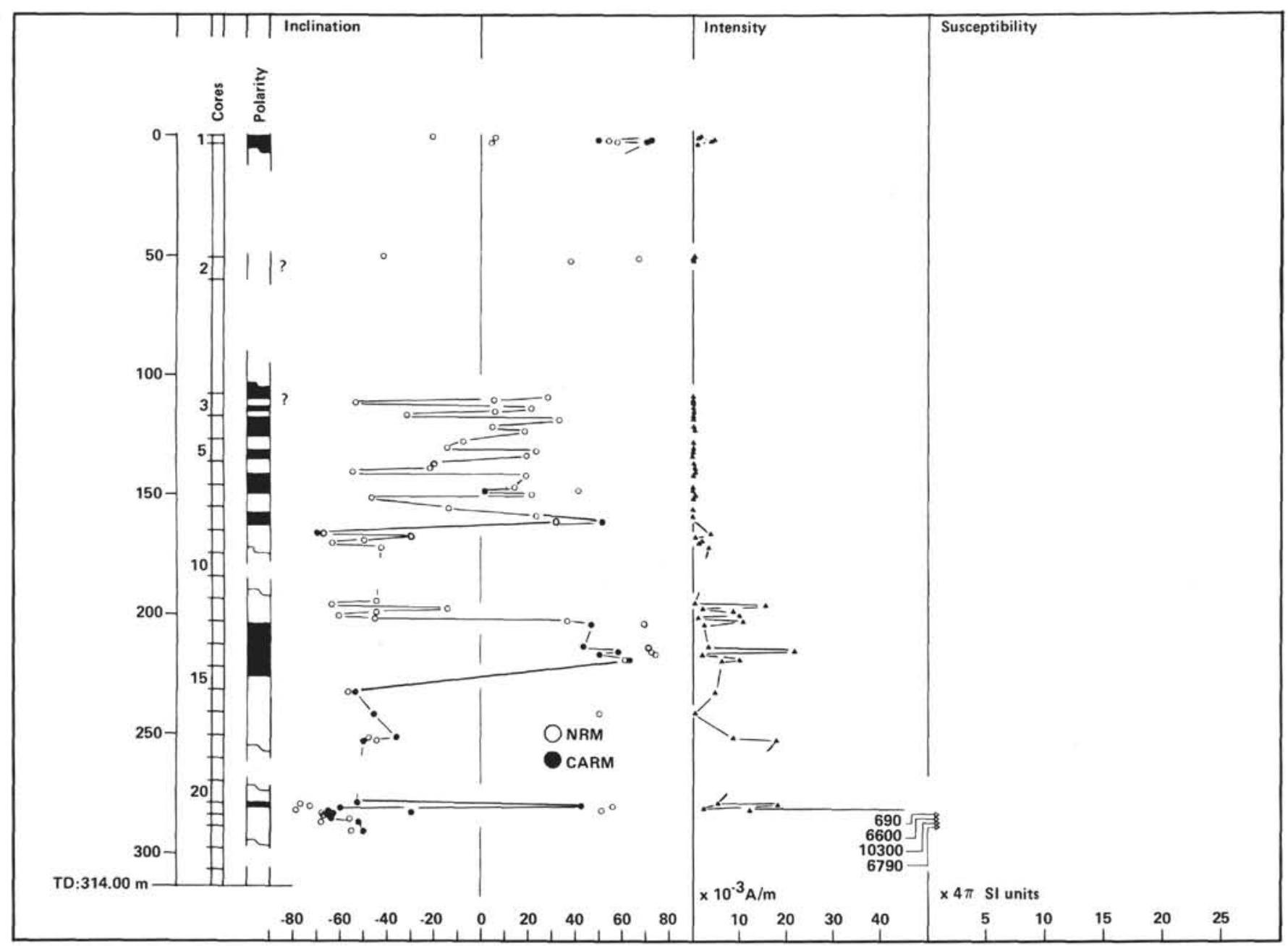

Figure 1. Paleomagnetics, Site 552. 


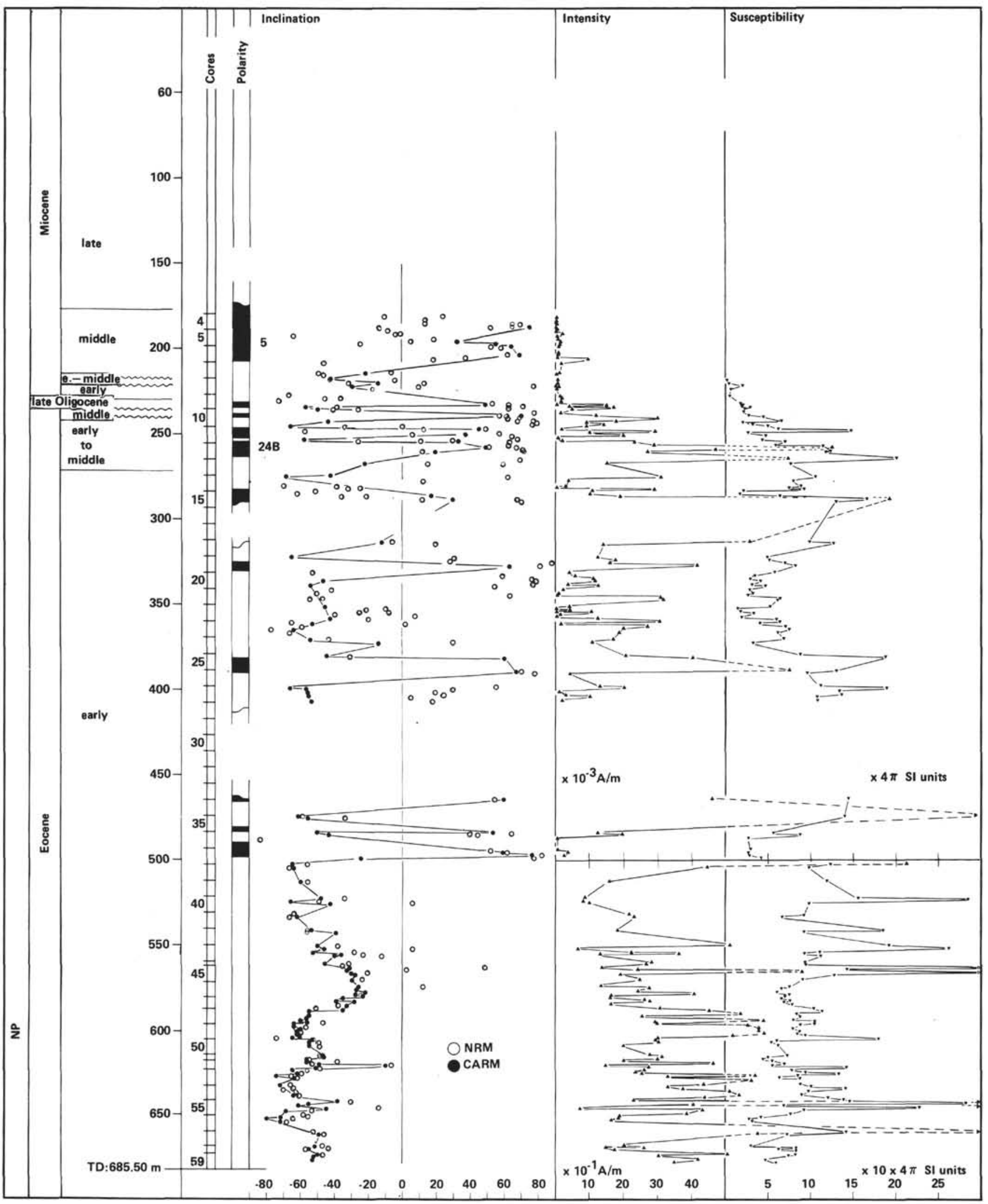

Figure 2. Paleomagnetics, Site 553. 


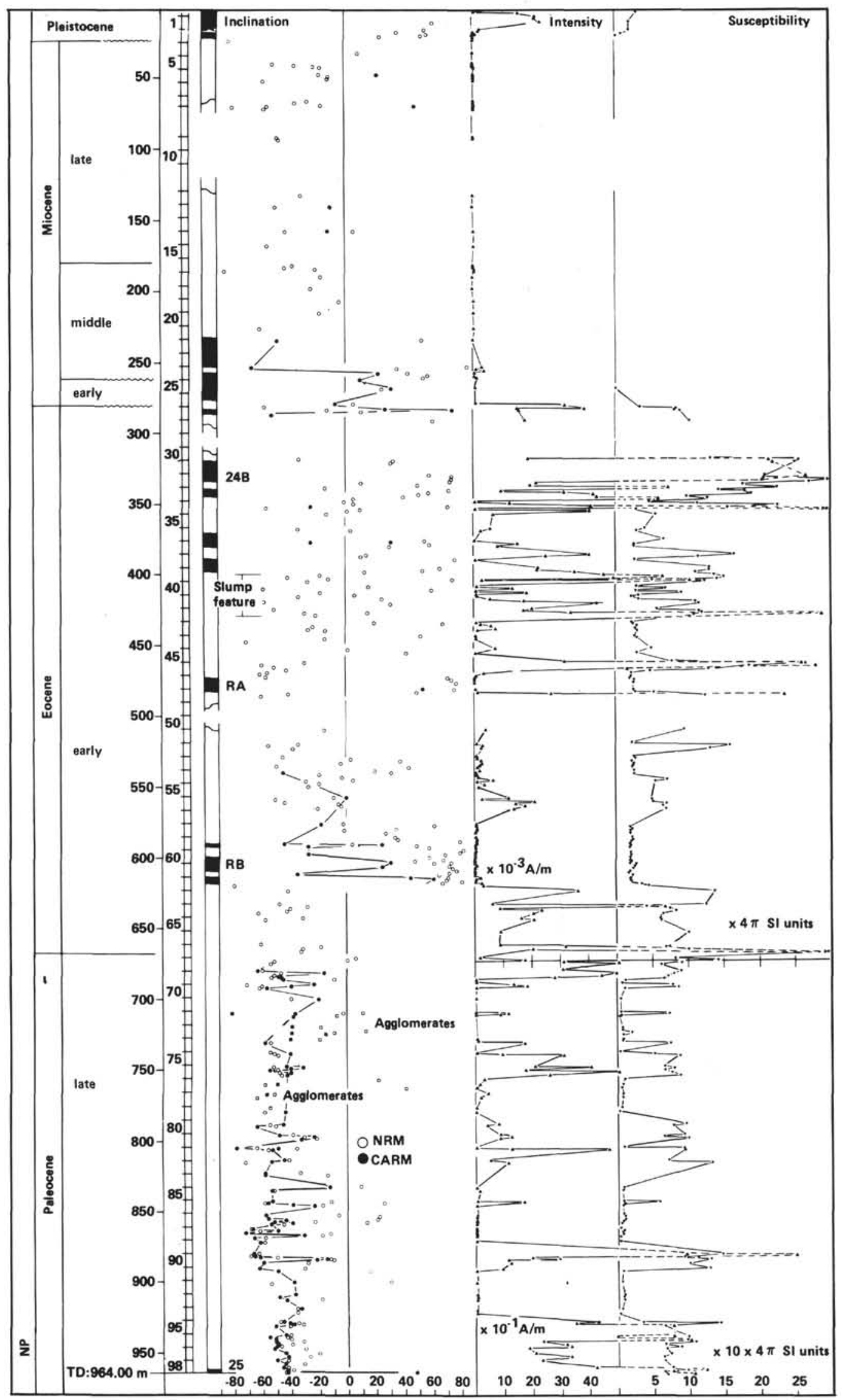

Figure 3. Paleomagnetics, Site 555. 